\title{
Observability-based Rules for Designing Consistent EKF SLAM Estimators
}

\author{
Guoquan P. Huang*, Anastasios I. Mourikis ${ }^{\dagger}$, and Stergios I. Roumeliotis* \\ *Dept. of Computer Science and Engineering, University of Minnesota, Minneapolis, MN 55455 \\ Email: \{ghuang|stergios\}@cs.umn.edu \\ ${ }^{\dagger}$ Dept. of Electrical Engineering, University of California, Riverside, CA 92521 \\ Email: mourikis@ee.ucr.edu
}

\begin{abstract}
In this work, we study the inconsistency problem of EKF-based SLAM from the perspective of observability. We analytically prove that when the Jacobians of the process and measurement models are evaluated at the latest state estimates during every time step, the linearized error-state system employed in the EKF has observable subspace of dimension higher than that of the actual, nonlinear, SLAM system. As a result, the covariance estimates of the EKF undergo reduction in directions of the state space where no information is available, which is a primary cause of the inconsistency. Based on these theoretical results, we propose a general framework for improving the consistency of EKF-based SLAM. In this framework, the EKF linearization points are selected in a way that ensures that the resulting linearized system model has an observable subspace of appropriate dimension. We describe two algorithms that are instances of this paradigm. In the first, termed Observability Constrained EKF (OC-EKF), the linearization points are selected so as to minimize their expected errors (i.e., the difference between the linearization point and the true state) under the observability constraints. In the second, the filter Jacobians are calculated using the first-ever available estimates for all state variables. This latter approach is termed First-Estimates Jacobian (FEJ)-EKF. The proposed algorithms have been tested both in simulation and experimentally, and are shown to significantly outperform the standard EKF both in terms of accuracy and consistency.
\end{abstract}

\section{INTRODUCTION}

Simultaneous localization and mapping (SLAM) is the process of building a map of an environment and concurrently generating an estimate of the robot pose (position and orientation) from the sensor readings. For autonomous vehicles exploring unknown environments, the ability to perform SLAM is essential. Since [Smith and Cheeseman, 1987] first introduced a stochastic-mapping solution to the SLAM problem, rapid and exciting progress has been made, resulting in several competing solutions. Recent interest in SLAM has focused on the design of estimation algorithms [Montemerlo, 2003], [Paskin, 2002], data association techniques [Neira and Tardos, 2001], and sensor data processing [Se et al., 2002]. Among the numerous algorithms developed thus far for the SLAM problem, the extended Kalman filter (EKF) remains one of the most popular ones, and has been used in several applications (e.g., [Newman, 1999], [Williams et al., 2000], [Kim and Sukkarieh, 2003]). However, in spite of its widespread adoption, the fundamental issue of the consistency of the EKFSLAM algorithm has not yet been sufficiently investigated.
As defined in [Bar-Shalom et al., 2001], a state estimator is consistent if the estimation errors are zero-mean and have covariance matrix smaller or equal to the one calculated by the filter. Consistency is one of the primary criteria for evaluating the performance of any estimator; if an estimator is inconsistent, then the accuracy of the produced state estimates is unknown, which in turn makes the estimator unreliable. Since SLAM is a nonlinear estimation problem, no provably consistent estimator can be constructed for it. The consistency of every estimator has to be evaluated experimentally. In particular for the standard EKF-SLAM algorithm, there exists significant empirical evidence showing that the computed state estimates tend to be inconsistent (cf. Section II).

In this paper, we investigate in depth one fundamental cause of the inconsistency of the standard EKF-SLAM algorithm. In particular, we revisit this problem from a new perspective, i.e., by analyzing the observability properties of the filter's system model. Our key conjecture in this paper is that the observability properties of the EKF linearized system model profoundly affect the performance of the filter, and are a significant factor in determining its consistency. Specifically, the major contributions of this work are the following:

- Through an observability analysis, we prove that the standard EKF-SLAM employs an error-state system model that has an unobservable subspace of dimension two, even though the underlying nonlinear system model has three unobservable degrees of freedom (corresponding to the position and orientation of the global reference frame). As a result, the filter gains spurious information along directions of the state space where no information is actually available. This leads to an unjustified reduction of the covariance estimates, and is a primary cause of filter inconsistency.

- Motivated by this analysis, we propose a new methodology for improving the consistency of EKF-based SLAM. Specifically, we propose selecting the linearization points of the EKF in a way that ensures that the unobservable subspace of the EKF system model is of appropriate dimension. In our previous work [Huang et al., 2008a], [Huang et al., 2008b], we proved that this can be achieved by computing the EKF Jacobians using the first-ever available estimates for each of the state variables. The resulting algorithm is termed First Estimates Jacobian 
(FEJ)-EKF. In this work, we propose an alternative approach, named Observability Constrained (OC)-EKF, which falls under the same general framework. In this novel filter, the EKF linearization points are selected so as not only to guarantee the desired observability properties but also to minimize the expected errors of the linearization points (i.e., the difference between the linearization point and the true state). This can be formulated as a constrained minimization problem, whose solution renders the linearization points used for computing the filter Jacobians.

- Through extensive simulations and real-world experiments, we verify that both the FEJ-EKF and the OCEKF outperform the standard EKF, even though they use less accurate linearization points in computing the filter Jacobians (since the linearization points used in the FEJ-EKF and OC-EKF are, in general, different from the latest, and thus best, state estimates). This result supports our conjecture that the observability properties of the EKF system model play a fundamental role in determining consistency.

The remainder of the paper is organized as follows. After an overview of related work in the next section, the standard EKF-SLAM formulation with generalized system and measurement models is described in Section III. In Section IV, the observability analysis of SLAM is presented and is employed to prove that the standard EKF-SLAM always has incorrect observability properties. Section V describes the proposed approaches for improving the consistency of EKF-SLAM, and in Sections VI and VII the performance of the FEJ-EKF and OC-EKF is demonstrated through Monte-Carlo simulations and experiments. Finally, Section VIII outlines the main conclusions of this work.

\section{RELATED WORK}

The inconsistency problem of the standard EKF-SLAM algorithm has recently attracted considerable interest [Castellanos et al., 2004], [Castellanos et al., 2007], [Julier and Uhlmann, 2001], [Bailey et al., 2006], [Huang and Dissanayake, 2006], [Huang and Dissanayake, 2007], [Huang et al., 2008a], [Huang et al., 2008b]. The first work to draw attention to this issue was that of [Julier and Uhlmann, 2001], who observed that when a stationary robot measures the relative position of a new landmark multiple times, the estimated variance of the robot's orientation becomes smaller. Since the observation of a previously unseen feature does not provide any information about the robot's state, this reduction is artificial, and leads to inconsistency. Additionally, a condition that the filter Jacobians need to satisfy in order to permit consistent estimation, was described. We show that this condition, derived in [Julier and Uhlmann, 2001] for the case of a stationary robot, is a special case of an observabilitybased condition derived in our work for the general case of a moving robot (cf. Lemma 5.1).

More recently, the work of [Huang and Dissanayake, 2007] extended the analysis of [Julier and Uhlmann, 2001] to the case of a robot that observes a landmark from two positions (i.e., the robot observes a landmark, moves and then re-observes the landmark). A constraint that the filter Jacobians need to fulfill in this case so as to allow for consistent estimation, was proposed. In [Huang and Dissanayake, 2007], it was shown that this condition is generally violated, due to the fact that the filter Jacobians at different time instants are evaluated using different estimates for the same state variables. Interestingly, in Section V-C it is shown that this condition can also be derived as a special case of our generalized analysis.

[Bailey et al., 2006] examined several symptoms of the inconsistency of the standard EKF-SLAM algorithm, and argued, based on Monte Carlo simulation results, that the uncertainty in the robot orientation is the main cause of the inconsistency of EKF-SLAM. However, no theoretical results were provided. The work of [Huang and Dissanayake, 2006] further confirmed the empirical findings in [Bailey et al., 2006], and argued by example that in EKF-SLAM the inconsistency is always in the form of overconfident estimates (i.e., the computed covariances are smaller than the actual ones).

The aforementioned works have described several symptoms of inconsistency that appear in the standard EKF-SLAM, and have analytically studied only a few special cases, such as that of a stationary robot [Julier and Uhlmann, 2001], and that of one-step motion [Huang and Dissanayake, 2007]. However, no theoretical analysis into the cause of inconsistency for the general case of a moving robot was conducted. To the best of our knowledge, the first such analysis appeared in our previous publications [Huang et al., 2008a], [Huang et al., 2008b]. Therein, the mismatch in the dimensions of the observable subspaces between the standard EKF and the underlying nonlinear SLAM system was identified as a fundamental cause of inconsistency, and the FEJ-EKF was proposed as a means of improving the consistency of the estimates.

In this paper, we present the theoretical analysis of [Huang et al., 2008a], [Huang et al., 2008b] in more detail, and propose a general framework for improving the consistency of EKFSLAM. It is shown that the FEJ-EKF is one of several possible estimators, which rely on the observability analysis for the selection of EKF linearization points. Moreover, we propose an alternative EKF estimator, the OC-EKF, whose performance is an improvement over the FEJ-EKF. The OC-EKF selects the optimal linearization points in a way that minimizes the the linearization errors, while ensuring that the observable subspace of the EKF linearized system model has correct dimensions. The following sections describe the theoretical development of the algorithms in detail.

\section{STANDARD EKF-SLAM FORMULATION}

In this section, we present the equations of the standard EKF-SLAM formulation with generalized system and measurement models. To preserve the clarity of the presentation, we first focus on the case where a single landmark is included in the state vector, while the case of multiple landmarks is addressed later on. In the standard formulation of SLAM, the state vector comprises the robot pose and the landmark position in the global frame of reference. Thus, at time-step $k$ 
the state vector is given by:

$$
\mathbf{x}_{k}=\left[\begin{array}{lll}
\mathbf{p}_{R_{k}}^{T} & \phi_{R_{k}} & \mathbf{p}_{L}^{T}
\end{array}\right]^{T}=\left[\begin{array}{ll}
\mathbf{x}_{R_{k}}^{T} & \mathbf{p}_{L}^{T}
\end{array}\right]^{T}
$$

where $\mathbf{x}_{R_{k}}=\left[\begin{array}{ll}\mathbf{p}_{R_{k}}^{T} & \phi_{R_{k}}\end{array}\right]^{T}$ denotes the robot pose, and $\mathbf{p}_{L}$ is the landmark position. EKF-SLAM recursively evolves in two steps: propagation and update, based on the discrete-time process and measurement models, respectively.

\section{A. EKF Propagation}

In the propagation step, the robot's odometry measurements are processed to obtain an estimate of the pose change between two consecutive time steps, and then employed in the EKF to propagate the robot state estimate. On the other hand, since the landmark is static, its state estimate does not change with the incorporation of a new odometry measurement. The EKF propagation equations are given by: ${ }^{1}$

$$
\begin{aligned}
\hat{\mathbf{p}}_{R_{k+1 \mid k}} & =\hat{\mathbf{p}}_{R_{k \mid k}}+\mathbf{C}\left(\hat{\phi}_{R_{k \mid k}}\right)^{R_{k}} \hat{\mathbf{p}}_{R_{k+1}} \\
\hat{\phi}_{R_{k+1 \mid k}} & =\hat{\phi}_{R_{k \mid k}}+{ }^{R_{k}} \hat{\phi}_{R_{k+1}} \\
\hat{\mathbf{p}}_{L_{k+1 \mid k}} & =\hat{\mathbf{p}}_{L_{k \mid k}}
\end{aligned}
$$

where $\mathbf{C}(\cdot)$ denotes the $2 \times 2$ rotation matrix, and ${ }^{R_{k}} \hat{\mathbf{x}}_{R_{k+1}}=$ $\left[{ }^{R_{k}} \hat{\mathbf{p}}_{R_{k+1}}^{T}{ }^{R_{k}} \hat{\phi}_{R_{k+1}}\right]^{T}$ is the odometry-based estimate of the robot's motion between time-steps $k$ and $k+1$. This estimate is corrupted by zero-mean, white Gaussian noise $\mathbf{w}_{k}={ }^{R_{k}} \mathbf{x}_{R_{k+1}}-{ }^{R_{k}} \hat{\mathbf{x}}_{R_{k+1}}$, with covariance matrix $\mathbf{Q}_{k}$. This process model is nonlinear, and can be described by the following generic nonlinear function:

$$
\mathbf{x}_{k+1}=\mathbf{f}\left(\mathbf{x}_{k},{ }^{R_{k}} \hat{\mathbf{x}}_{R_{k+1}}+\mathbf{w}_{k}\right)
$$

In addition to the state propagation equations, the linearized error-state propagation equation is necessary for the EKF. This is given by:

$$
\begin{aligned}
\tilde{\mathbf{x}}_{k+1 \mid k} & =\left[\begin{array}{cc}
\boldsymbol{\Phi}_{R_{k}} & \mathbf{0}_{3 \times 2} \\
\mathbf{0}_{2 \times 3} & \mathbf{I}_{2}
\end{array}\right]\left[\begin{array}{c}
\tilde{\mathbf{x}}_{R_{k \mid k}} \\
\tilde{\mathbf{p}}_{L_{k \mid k}}
\end{array}\right]+\left[\begin{array}{c}
\mathbf{G}_{R_{k}} \\
\mathbf{0}_{2 \times 2}
\end{array}\right] \mathbf{w}_{k} \\
& \triangleq \boldsymbol{\Phi}_{k} \tilde{\mathbf{x}}_{k \mid k}+\mathbf{G}_{k} \mathbf{w}_{k}
\end{aligned}
$$

where $\boldsymbol{\Phi}_{R_{k}}$ and $\mathbf{G}_{R_{k}}$ are obtained from the state propagation equations (2)-(3):

$$
\begin{aligned}
\boldsymbol{\Phi}_{R_{k}} & =\left[\begin{array}{cc}
\mathbf{I}_{2} & \mathbf{J C}\left(\hat{\phi}_{R_{k \mid k}}\right)^{R_{k}} \hat{\mathbf{p}}_{R_{k+1}} \\
\mathbf{0}_{1 \times 2} & 1
\end{array}\right] \\
& \equiv\left[\begin{array}{cc}
\mathbf{I}_{2} & \mathbf{J}\left(\hat{\mathbf{p}}_{R_{k+1 \mid k}}-\hat{\mathbf{p}}_{R_{k \mid k}}\right) \\
\mathbf{0}_{1 \times 2} & 1
\end{array}\right] \\
\mathbf{G}_{R_{k}} & =\left[\begin{array}{cc}
\mathbf{C}\left(\hat{\phi}_{R_{k \mid k}}\right) & \mathbf{0}_{2 \times 1} \\
\mathbf{0}_{1 \times 2} & 1
\end{array}\right]
\end{aligned}
$$

with $\mathbf{J} \triangleq\left[\begin{array}{cc}0 & -1 \\ 1 & 0\end{array}\right]$.

It is important to point out that the form of the propagation equations presented above is general, and holds for any robot

\footnotetext{
${ }^{1}$ Throughout this paper the subscript $\ell \mid j$ refers to the estimate of a quantity at time-step $\ell$, after all measurements up to time-step $j$ have been processed. $\hat{x}$ is used to denote the estimate of a random variable $x$, while $\tilde{x}=x-\hat{x}$ is the error in this estimate. $\mathbf{0}_{m \times n}$ and $\mathbf{1}_{m \times n}$ denote $m \times n$ matrices of zeros and ones, respectively, while $\mathbf{I}_{n}$ is the $n \times n$ identity matrix. Finally, we use the concatenated forms $s \phi$ and $c \phi$ to denote the $\sin \phi$ and $\cos \phi$ functions, respectively.
}

kinematic model (e.g., unicycle, bicycle, or Ackerman model). In Appendix A, we derive the expressions for (2)-(4), as well as the state and noise Jacobians, for the common case where the unicycle model is used.

\section{B. EKF Update}

During SLAM, the measurement used for updates in the EKF is a function of the relative position of the landmark with respect to the robot:

$$
\mathbf{z}_{k}=\mathbf{h}\left(\mathbf{x}_{k}\right)+\mathbf{v}_{k}=\mathbf{h}\left({ }^{R_{k}} \mathbf{p}_{L}\right)+\mathbf{v}_{k}
$$

where ${ }^{R_{k}} \mathbf{p}_{L}=\mathbf{C}^{T}\left(\phi_{R_{k}}\right)\left(\mathbf{p}_{L}-\mathbf{p}_{R_{k}}\right)$ is the position of the landmark with respect to the robot at time-step $k$, and $\mathbf{v}_{k}$ is zero-mean Gaussian measurement noise with covariance $\mathbf{R}_{k}$. In this work, we allow $\mathbf{h}$ to be any measurement function. For instance, $\mathbf{z}_{k}$ can be a direct measurement of relative position, a pair of range and bearing measurements, bearing-only measurements from monocular cameras, etc. Generally, the measurement function is nonlinear, and hence it is linearized for use in the EKF. The linearized measurement error equation is given by:

$$
\begin{aligned}
\tilde{\mathbf{z}}_{k} & \simeq\left[\begin{array}{ll}
\mathbf{H}_{R_{k}} & \mathbf{H}_{L_{k}}
\end{array}\right]\left[\begin{array}{c}
\tilde{\mathbf{x}}_{R_{k \mid k-1}} \\
\tilde{\mathbf{p}}_{L_{k \mid k-1}}
\end{array}\right]+\mathbf{v}_{k} \\
& \triangleq \mathbf{H}_{k} \tilde{\mathbf{x}}_{k \mid k-1}+\mathbf{v}_{k}
\end{aligned}
$$

where $\mathbf{H}_{R_{k}}$ and $\mathbf{H}_{L_{k}}$ are the Jacobians of $\mathbf{h}$ with respect to the robot pose and the landmark position, respectively, evaluated at the state estimate $\hat{\mathbf{x}}_{k \mid k-1}$. Using the chain rule of differentiation, these are computed as:

$$
\begin{aligned}
& \mathbf{H}_{R_{k}}=\left(\nabla \mathbf{h}_{k}\right) \mathbf{C}^{T}\left(\hat{\phi}_{R_{k \mid k-1}}\right)\left[\begin{array}{ll}
-\mathbf{I}_{2} & -\mathbf{J}\left(\hat{\mathbf{p}}_{L_{k \mid k-1}}-\hat{\mathbf{p}}_{R_{k \mid k-1}}\right)
\end{array}\right] \\
& \mathbf{H}_{L_{k}}=\left(\nabla \mathbf{h}_{k}\right) \mathbf{C}^{T}\left(\hat{\phi}_{R_{k \mid k-1}}\right)
\end{aligned}
$$

where $\nabla \mathbf{h}_{k}$ denotes the Jacobian of $\mathbf{h}$ with respect to the robot-relative landmark position (i.e., with respect to the vector ${ }^{R} \mathbf{p}_{L}$ ), evaluated at the state estimate $\hat{\mathbf{x}}_{k \mid k-1}$.

\section{SLAM OBSERVABILITY ANALYSIS}

In this section, we perform an observability analysis for the generalized EKF-SLAM formulation derived in the previous section, and compare its properties with those of the underlying nonlinear system. Based on this analysis, we draw conclusions about the consistency of the filter. We note that, to keep the presentation clear, some intermediate steps of the derivations have been omitted. The interested reader is referred to [Huang et al., 2008c] for details.

It should be pointed out that the observability properties of SLAM have been studied in only a few cases in the literature. In particular, [Andrade-Cetto and Sanfeliu, 2004], [AndradeCetto and Sanfeliu, 2005] investigated the observability of a simple linear time-invariant (LTI) SLAM system, and showed that it is unobservable. The work of [Vidal-Calleja et al., 2007] approximated the SLAM system by a piecewise constant linear (PWCL) one, and applied the technique of [GoshenMeskin and Bar-Itzhack, 1992] to study the observability properties of bearing-only SLAM. On the other hand, in [Lee 
et al., 2006], [Huang et al., 2008a] the observability properties of the nonlinear SLAM system were studied using the nonlinear observability rank condition introduced by [Hermann and Krener, 1977]. These works proved that the nonlinear SLAM system is unobservable, with three unobservable degrees of freedom.

All the aforementioned approaches examine the observability properties of the nonlinear SLAM system, or of linear approximations to it. However, to the best of our knowledge, an analysis of the observability properties of the EKF linearized error-state system model had not been carried out prior to our work [Huang et al., 2008a], [Huang et al., 2008b]. Since this model is the one used in any actual EKF implementation, a lack of understanding of its observability properties appears to be a significant limitation. In fact, as shown in this paper, these properties play a significant role in determining the consistency of the filter, and form the basis of our approach for improving the performance of the estimator.

\section{A. Nonlinear Observability Analysis for SLAM}

We start by carrying out the observability analysis for the continuous-time nonlinear SLAM system. This analysis is based on the observability rank condition introduced in [Hermann and Krener, 1977]. Specifically, Theorem 3.11 therein states that "if a nonlinear system is locally weakly observable, the observability rank condition is satisfied generically". We here show that the SLAM system does not satisfy the observability rank condition, and thus it is not locally weakly observable nor locally observable. In particular, we conduct the analysis for a general measurement model, instead of only relative-position or distance-bearing measurements as in [Huang et al., 2008a], [Lee et al., 2006].

For the continuous-time analysis, we employ a unicycle kinematic model, although similar conclusions can be drawn if different models are used [Lee et al., 2006]. The process model in continuous-time form is given by:

$$
\begin{aligned}
{\left[\begin{array}{c}
\dot{x}_{R}(t) \\
\dot{y}_{R}(t) \\
\dot{\phi}_{R}(t) \\
\dot{x}_{L}(t) \\
\dot{y}_{L}(t)
\end{array}\right] } & =\left[\begin{array}{c}
c \phi_{R}(t) \\
s \phi_{R}(t) \\
0 \\
0 \\
0
\end{array}\right] v(t)+\left[\begin{array}{l}
0 \\
0 \\
1 \\
0 \\
0
\end{array}\right] \omega(t) \\
\Rightarrow \dot{\mathbf{x}}(t) & =\mathbf{f}_{1} v(t)+\mathbf{f}_{2} \omega(t)
\end{aligned}
$$

where $\mathbf{u} \triangleq\left[\begin{array}{ll}v & \omega\end{array}\right]^{T}$ is the control input, consisting of linear and rotational velocity. Since any type of measurement during SLAM is a function of the relative position of the landmark with respect to the robot, we can write the measurement model in the following generic form:

$$
\begin{aligned}
z(t) & =h(\rho, \psi) \\
\rho & =\left\|\mathbf{p}_{L}-\mathbf{p}_{R}\right\| \\
\psi & =\operatorname{atan} 2\left(y_{L}-y_{R}, x_{L}-x_{R}\right)-\phi_{R}
\end{aligned}
$$

where $\rho$ and $\psi$ are the robot-to-landmark relative distance and bearing angle, respectively. Note that parameterizing the measurement with respect to $\rho$ and $\psi$ is equivalent to parameterizing it with respect to the relative landmark position expressed in the robot frame, ${ }^{R} \mathbf{p}_{L}$. The relation between these quantities is ${ }^{R} \mathbf{p}_{L}=\rho\left[\begin{array}{l}c \psi \\ s \psi\end{array}\right]$. The analysis will be based on the following lemma:

Lemma 4.1: All the Lie derivatives of the nonlinear SLAM system (cf. (14) and (15)) are functions of $\rho$ and $\psi$ only.

Proof: See Appendix B

We will now employ this result for the nonlinear observability analysis. In particular, assume that a number of different measurements are available, $z_{i}=h_{i}(\rho, \psi), i=1,2, \ldots, n$. Then, since all the Lie derivatives for all measurements are functions of $\rho$ and $\psi$ only, we can prove the following:

Lemma 4.2: The space spanned by all the $k$-th order Lie derivatives $L_{\mathbf{f}_{j}}^{k} h_{i}(\forall k \in \mathbb{N}, j=1,2, i=1,2, \ldots, n)$ is denoted by $\mathcal{G}$, and the space $d \mathcal{G}$ spanned by the gradients of the elements of $\mathcal{G}$ is given by:

$d \mathcal{G}=\underset{\text { row }}{\operatorname{span}}\left[\begin{array}{ccccc}s \phi_{R} & -c \phi_{R} & -c \phi_{R} \delta x-s \phi_{R} \delta y & -s \phi_{R} & c \phi_{R} \\ c \phi_{R} & s \phi_{R} & s \phi_{R} \delta x-c \phi_{R} \delta y & -c \phi_{R} & -s \phi_{R}\end{array}\right]$

where $\delta x \triangleq x_{L}-x_{R}$ and $\delta y \triangleq y_{L}-y_{R}$.

Proof: See Appendix C

The matrix shown above is the "observability matrix" for the nonlinear SLAM system under consideration. Clearly, this is not a full-rank matrix, and the system is unobservable. Intuitively, this is a consequence of the fact that we cannot gain absolute, but rather only relative state information from the available measurements. Even though the notion of an "unobservable subspace" cannot be strictly defined for this system, the physical interpretation of the basis of $d \mathcal{G}^{\perp}$ will give us useful information for our analysis in Section IV-B. By inspection, we see that one possible basis for the space $d \mathcal{G}^{\perp}$ is given by:

$$
d \mathcal{G}^{\perp}=\underset{\operatorname{span}}{\operatorname{col} .}\left[\begin{array}{ccc}
1 & 0 & -y_{R} \\
0 & 1 & x_{R} \\
0 & 0 & 1 \\
1 & 0 & -y_{L} \\
0 & 1 & x_{L}
\end{array}\right] \triangleq \operatorname{span}\left[\begin{array}{lll}
\mathbf{n}_{1} & \mathbf{n}_{2} & \mathbf{n}_{3}
\end{array}\right]
$$

From the structure of the vectors $\mathbf{n}_{1}$ and $\mathbf{n}_{2}$ we see that a change in the state by $\Delta \mathbf{x}=\alpha \mathbf{n}_{1}+\beta \mathbf{n}_{2}, \alpha, \beta \in \mathbb{R}$ corresponds to a "shifting" of the $x-y$ plane by $\alpha$ units along $x$, and by $\beta$ units along $y$. Thus, if the robot and landmark positions are shifted equally, the states $\mathrm{x}$ and $\mathrm{x}+\Delta \mathrm{x}$ will be indistinguishable given the measurements. To understand the physical meaning of $\mathbf{n}_{3}$, we consider the case where the $x-y$ plane is rotated by a small angle $\delta \phi$. Rotating the coordinate system transforms any point $\mathbf{p}=\left[\begin{array}{ll}x & y\end{array}\right]^{T}$ to a point $\mathbf{p}^{\prime}=\left[\begin{array}{ll}x^{\prime} & y^{\prime}\end{array}\right]^{T}$, given by:

$$
\left[\begin{array}{l}
x^{\prime} \\
y^{\prime}
\end{array}\right]=\mathbf{C}(\delta \phi)\left[\begin{array}{l}
x \\
y
\end{array}\right] \simeq\left[\begin{array}{cc}
1 & -\delta \phi \\
\delta \phi & 1
\end{array}\right]\left[\begin{array}{l}
x \\
y
\end{array}\right]=\left[\begin{array}{l}
x \\
y
\end{array}\right]+\delta \phi\left[\begin{array}{c}
-y \\
x
\end{array}\right]
$$

where we have employed the small angle approximations $c(\delta \phi) \simeq 1$ and $s(\delta \phi) \simeq \delta \phi$. Using this result, we see that if the plane containing the robot and landmarks is rotated by 
$\delta \phi$, the SLAM state vector will change to:

$$
\mathbf{x}^{\prime}=\left[\begin{array}{c}
x_{R}^{\prime} \\
y_{R}^{\prime} \\
\phi_{R}^{\prime} \\
x_{L}^{\prime} \\
y_{L}^{\prime}
\end{array}\right] \simeq\left[\begin{array}{l}
x_{R} \\
y_{R} \\
\phi_{R} \\
x_{L} \\
y_{L}
\end{array}\right]+\delta \phi\left[\begin{array}{c}
-y_{R} \\
x_{R} \\
1 \\
-y_{L} \\
x_{L}
\end{array}\right]=\mathbf{x}+\delta \phi \mathbf{n}_{3}
$$

which indicates that the vector $\mathbf{n}_{3}$ corresponds to a rotation of the $x-y$ plane. Since $\mathbf{n}_{3} \in d \mathcal{G}^{\perp}$, this result shows that any such rotation is unobservable, and will cause no change to the measurements. The preceding analysis for the meaning of the basis vectors of $d \mathcal{G}^{\perp}$ agrees with intuition, which dictates that the global coordinates of the state vector in SLAM (rotation and translation) are unobservable.

\section{B. EKF-SLAM Observability Analysis}

In the previous section, it was shown that the underlying physical system in SLAM has three unobservable degrees of freedom. Thus, when the EKF is used for state estimation in SLAM, we would expect that the system model employed by the EKF also shares this property. However, in this section we show that this is not the case, since the unobservable subspace of the linearized error-state model of the standard EKF is generally of dimension only 2 .

First recall that in general the Jacobian matrices $\mathbf{\Phi}_{k}, \mathbf{G}_{k}$, and $\mathbf{H}_{k}$ used in the EKF-SLAM linearized error-state model (cf. (6) and (11)), are defined as:

$$
\begin{aligned}
\mathbf{\Phi}_{k} & =\left.\nabla_{\mathbf{x}_{k}} \mathbf{f}\right|_{\left\{\mathbf{x}_{k \mid k}^{\star}, \mathbf{x}_{k+1 \mid k}^{\star}, \mathbf{0}\right\}} \\
\mathbf{G}_{k} & =\left.\nabla_{\mathbf{w}_{k}} \mathbf{f}\right|_{\left\{\mathbf{x}_{k \mid k}^{\star}, \mathbf{0}\right\}} \\
\mathbf{H}_{k} & =\left.\nabla_{\mathbf{x}_{k}} \mathbf{h}\right|_{\mathbf{x}_{k \mid k-1}^{\star}}
\end{aligned}
$$

In these expressions, $\mathbf{x}_{\ell \mid \ell-1}^{\star}$ and $\mathbf{x}_{\ell \mid \ell}^{\star}(\ell=k, k+1)$ denote the linearization points for the state $\mathbf{x}_{\ell}$, used for evaluating the Jacobians before and after the EKF update at time-step $\ell$, respectively. A linearization point equal to the zero vector is chosen for the noise. The EKF employs the above linearized system model for propagating and updating the estimates of the state vector and covariance matrix, and thus the observability properties of this model affect the performance of the estimator. To the best of our knowledge, a study of these properties has not been carried out in the past, and is one of the main contributions of this work.

Since the linearized error-state model for EKF-SLAM is time-varying, we employ the local observability matrix [Chen et al., 1990] to perform the observability analysis. Specifically, the local observability matrix for the time interval between time-steps $k_{o}$ and $k_{o}+m$ is defined as:

$$
\begin{aligned}
\mathbf{M} & \triangleq\left[\begin{array}{c}
\mathbf{H}_{k_{o}} \\
\mathbf{H}_{k_{o}+1} \boldsymbol{\Phi}_{k_{o}} \\
\vdots \\
\mathbf{H}_{k_{o}+m} \boldsymbol{\Phi}_{k_{o}+m-1} \cdots \boldsymbol{\Phi}_{k_{o}}
\end{array}\right] \\
& =\left[\begin{array}{cc}
\mathbf{H}_{R_{k_{o}}} & \mathbf{H}_{L_{k_{o}}} \\
\mathbf{H}_{R_{k_{o}+1}} \boldsymbol{\Phi}_{R_{k_{o}}} & \mathbf{H}_{L_{k_{o}+1}} \\
\vdots & \vdots \\
\mathbf{H}_{R_{k_{o}+m}} \boldsymbol{\Phi}_{R_{k_{o}+m-1}} \ldots \boldsymbol{\Phi}_{R_{k_{o}}} & \mathbf{H}_{L_{k_{o}+m}}
\end{array}\right] \\
& =\mathbf{M}\left(\mathbf{x}_{k_{o} \mid k_{o}-1}^{\star}, \mathbf{x}_{k_{o} \mid k_{o}}^{\star}, \ldots, \mathbf{x}_{k_{o}+m \mid k_{o}+m-1}, \mathbf{x}_{k_{o}+m \mid k_{o}+m}^{\star}\right)
\end{aligned}
$$

where (24) is obtained by substituting the matrices $\boldsymbol{\Phi}_{k}$ and $\mathbf{H}_{k}$ (cf. (6) and (11), respectively) into (23). The last expression, (25), makes explicit the fact that the observability matrix is a function of the linearization points used in computing all the Jacobians within the time interval $\left[k_{o}, k_{o}+m\right]$. In turn, this implies that the choice of linearization points affects the observability properties of the linearized error-state system of the EKF. This key fact is the basis of our analysis. In the following, we discuss different possible choices for linearization, and the observability properties of the corresponding linearized systems.

1) Ideal EKF-SLAM: Before considering the rank of the matrix $\mathbf{M}$, which is constructed using the estimated values of the state in the filter Jacobians, it is interesting to study the observability properties of the "oracle", or "ideal" EKF (i.e., the filter whose Jacobians are evaluated using the true values of the state variables, in other words, $\mathbf{x}_{k \mid k-1}^{\star}=\mathbf{x}_{k \mid k}^{\star}=\mathbf{x}_{k}$, for all $k$ ). In the following, all matrices evaluated using the true state values are denoted by the symbol " $\cup$ ".

We start by noting that (cf. (8)):

$$
\breve{\mathbf{\Phi}}_{R_{k_{o}+1}} \breve{\mathbf{\Phi}}_{R_{k_{o}}}=\left[\begin{array}{cc}
\mathbf{I}_{2} & \mathbf{J}\left(\mathbf{p}_{R_{k_{o}+2}}-\mathbf{p}_{R_{k_{o}}}\right) \\
\mathbf{0}_{1 \times 2} & 1
\end{array}\right]
$$

Based on this property, it is easy to show by induction that:

$\breve{\mathbf{\Phi}}_{R_{k_{o}+\ell-1}} \breve{\mathbf{\Phi}}_{R_{k_{o}+\ell-2}} \ldots \breve{\mathbf{\Phi}}_{R_{k_{o}}}=\left[\begin{array}{cc}\mathbf{I}_{2} & \mathbf{J}\left(\mathbf{p}_{R_{k_{o}+\ell}}-\mathbf{p}_{R_{k_{o}}}\right) \\ \mathbf{0}_{1 \times 2} & 1\end{array}\right]$

which holds for all $\ell>0$. Using this result, and substituting for the measurement Jacobians from (12) and (13), we can prove the following useful identity:

$$
\begin{aligned}
& \breve{\mathbf{H}}_{R_{k_{o}+\ell}} \breve{\mathbf{\Phi}}_{R_{k_{o}+\ell-1}} \cdots \breve{\mathbf{\Phi}}_{R_{k_{o}}}
\end{aligned}
$$

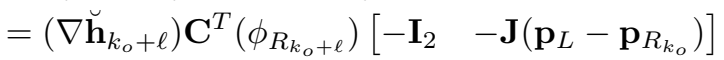

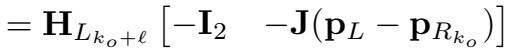

which holds for all $\ell>0$. The observability matrix $\breve{M}$ can 
now be written as:

$$
\begin{aligned}
\breve{\mathbf{M}} & =\underbrace{\operatorname{Diag}\left(\breve{\mathbf{H}}_{L_{k_{o}}}, \breve{\mathbf{H}}_{L_{k_{o}+1}}, \cdots, \breve{\mathbf{H}}_{L_{k_{o}+m}}\right)}_{\breve{\mathbf{D}}} \\
& \times \underbrace{\left[\begin{array}{ccc}
-\mathbf{I}_{2} & -\mathbf{J}\left(\mathbf{p}_{L}-\mathbf{p}_{R_{k_{o}}}\right) & \mathbf{I}_{2} \\
-\mathbf{I}_{2} & -\mathbf{J}\left(\mathbf{p}_{L}-\mathbf{p}_{R_{k_{o}}}\right) & \mathbf{I}_{2} \\
\vdots & \vdots & \vdots \\
-\mathbf{I}_{2} & -\mathbf{J}\left(\mathbf{p}_{L}-\mathbf{p}_{R_{k_{o}}}\right) & \mathbf{I}_{2}
\end{array}\right]}_{\breve{\mathbf{N}}}
\end{aligned}
$$

Lemma 4.3: The rank of the observability matrix, $\breve{M}$, of the ideal EKF is 2.

Proof: The rank of the product of the matrices $\breve{\mathbf{D}}$ and $\breve{\mathbf{N}}$ is given by (cf. (4.5.1) in [Meyer, 2001]):

$$
\operatorname{rank}(\breve{\mathbf{D}} \breve{\mathbf{N}})=\operatorname{rank}(\breve{\mathbf{N}})-\operatorname{dim}(\mathcal{N}(\breve{\mathbf{D}}) \bigcap \mathcal{R}(\breve{\mathbf{N}}))
$$

Since $\breve{\mathbf{N}}$ comprises $m+1$ repetitions of the same $2 \times 5$ block row, it is clear that $\operatorname{rank}(\breve{\mathbf{N}})=2$, and the range of $\breve{\mathbf{N}}, \mathcal{R}(\breve{\mathbf{N}})$, is spanned by the vectors $\mathbf{u}_{1}$ and $\mathbf{u}_{2}$, defined as follows:

$$
\left[\begin{array}{ll}
\mathbf{u}_{1} & \mathbf{u}_{2}
\end{array}\right]=\left[\begin{array}{c}
\mathbf{I}_{2} \\
\vdots \\
\mathbf{I}_{2}
\end{array}\right]
$$

We now observe that in general $\breve{\mathbf{D}} \mathbf{u}_{i} \neq \mathbf{0}$, for $i=1,2$. Moreover, note that any vector $\mathbf{y} \in \mathcal{R}(\breve{\mathbf{N}}) \backslash \mathbf{0}$ can be written as $\mathbf{y}=\alpha_{1} \mathbf{u}_{1}+\alpha_{2} \mathbf{u}_{2}$ for some $\alpha_{1}, \alpha_{2} \in \mathbb{R}$, where $\alpha_{1}$ and $\alpha_{2}$ are not simultaneously equal to zero. Thus, we see that in general $\breve{\mathbf{D}} \mathbf{y}=\alpha_{1} \breve{\mathbf{D}} \mathbf{u}_{1}+\alpha_{2} \breve{\mathbf{D}} \mathbf{u}_{2} \neq \mathbf{0}$, which implies that $\mathbf{y}$ does not belong to the nullspace $\mathcal{N}(\breve{\mathbf{D}})$ of $\breve{\mathbf{D}}$. Therefore, $\operatorname{dim}(\mathcal{N}(\breve{\mathbf{D}}) \cap \mathcal{R}(\breve{\mathbf{N}}))=0$, and, finally, $\operatorname{rank}(\breve{\mathbf{M}})=$ $\operatorname{rank}(\breve{\mathbf{N}})-\operatorname{dim}(\mathcal{N}(\breve{\mathbf{D}}) \cap \mathcal{R}(\breve{\mathbf{N}}))=\operatorname{rank}(\breve{\mathbf{N}})=2$.

Most importantly, it can be easily verified that a basis for the right nullspace of $\mathbf{N}$ (and thus for the right nullspace of $\mathbf{M}$ ) is given by the vectors shown in (18). Thus, the unobservable subspace of the ideal EKF system model is identical to the space $d \mathcal{G}^{\perp}$, which contains the unobservable directions of the nonlinear SLAM system. We therefore see that if it was possible to evaluate the Jacobians using the true state values, the linearized error-state model employed in the EKF would have observability properties similar to those of the actual, nonlinear SLAM system.

The preceding analysis was carried out for the case where a single landmark is included in the state vector. We now examine the more general case where $M>1$ landmarks are included in the state. Suppose the $M$ landmarks are observed at time-step $k_{o}+\ell(\ell>0)$, then the measurement matrix $\breve{\mathbf{H}}_{k_{o}+\ell}$ is given by: ${ }^{2}$

$$
\breve{\mathbf{H}}_{k_{o}+\ell}=\left[\begin{array}{cccc}
\breve{\mathbf{H}}_{R_{k_{o}+\ell}}^{(1)} & \breve{\mathbf{H}}_{L_{k_{o}+\ell}}^{(1)} & \cdots & \mathbf{0} \\
\vdots & \vdots & \ddots & \vdots \\
\breve{\mathbf{H}}_{R_{k_{o}+\ell}}^{(M)} & \mathbf{0} & \cdots & \breve{\mathbf{H}}_{L_{k_{o}+\ell}}^{(M)}
\end{array}\right]
$$

\footnotetext{
${ }^{2}$ We here assume that all $M$ landmarks are observed at every time step in the time interval $\left[k_{o}, k_{o}+m\right]$. This is done only to simplify the notation, and is not a necessary assumption in the analysis.
}

where $\breve{\mathbf{H}}_{R_{k_{o}+\ell}}^{(i)}$ and $\breve{\mathbf{H}}_{L_{k_{o}+\ell}}^{(i)}(i=1,2, \ldots, M)$, are obtained by (12) and (13) using the true values of the states, respectively. The observability matrix $\breve{M}$ now becomes:

$$
\begin{aligned}
& \breve{M}=
\end{aligned}
$$

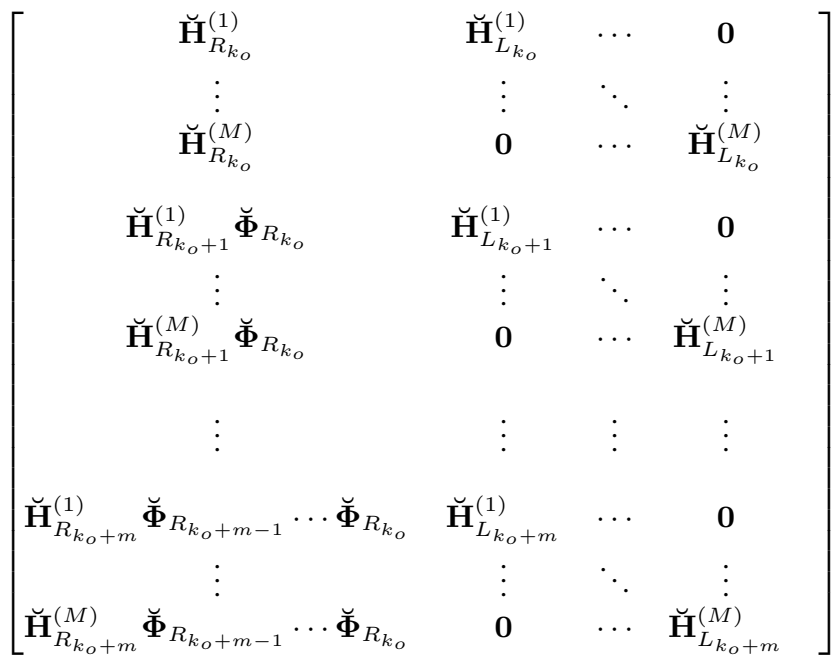

Using the identity (27), substitution of the Jacobian matrices in (32) yields:

$$
\begin{aligned}
& \breve{\mathbf{M}}=\underbrace{\operatorname{Diag}\left(\breve{\mathbf{H}}_{L_{k_{o}}}^{(1)}, \cdots, \breve{\mathbf{H}}_{L_{k_{o}+m}}^{(M)}\right)}_{\breve{\mathbf{D}}} \\
& \times \underbrace{\left[\begin{array}{ccccc}
-\mathbf{I}_{2} & -\mathbf{J}\left(\mathbf{p}_{L_{1}}-\mathbf{p}_{R_{k_{o}}}\right) & \mathbf{I}_{2} & \cdots & \mathbf{0}_{2 \times 2} \\
\vdots & \vdots & \vdots & \ddots & \vdots \\
-\mathbf{I}_{2} & -\mathbf{J}\left(\mathbf{p}_{L_{M}}-\mathbf{p}_{R_{k_{o}}}\right) & \mathbf{0}_{2 \times 2} & \cdots & \mathbf{I}_{2} \\
-\mathbf{I}_{2} & -\mathbf{J}\left(\mathbf{p}_{L_{1}}-\mathbf{p}_{R_{k_{o}}}\right) & \mathbf{I}_{2} & \cdots & \mathbf{0}_{2 \times 2} \\
\vdots & \vdots & \vdots & \ddots & \vdots \\
-\mathbf{I}_{2} & -\mathbf{J}\left(\mathbf{p}_{L_{M}}-\mathbf{p}_{R_{k_{o}}}\right) & \mathbf{0}_{2 \times 2} & \cdots & \mathbf{I}_{2} \\
\vdots & \vdots & \vdots & \vdots & \vdots \\
-\mathbf{I}_{2} & -\mathbf{J}\left(\mathbf{p}_{L_{1}}-\mathbf{p}_{R_{k_{o}}}\right) & \mathbf{I}_{2} & \cdots & \mathbf{0}_{2 \times 2} \\
\vdots & \vdots & \vdots & \ddots & \vdots \\
-\mathbf{I}_{2} & -\mathbf{J}\left(\mathbf{p}_{L_{M}}-\mathbf{p}_{R_{k_{o}}}\right) & \mathbf{0}_{2 \times 2} & \cdots & \mathbf{I}_{2}
\end{array}\right]}
\end{aligned}
$$

Clearly, the matrix $\breve{\mathbf{N}}$ now consists of $m+1$ repetitions of the $M$ block rows:

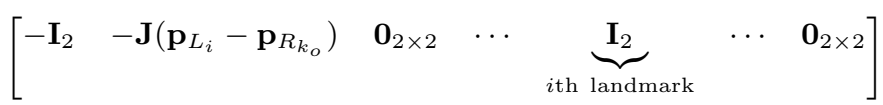

for $i=1,2, \ldots, M$. Therefore, $\operatorname{rank}(\breve{\mathbf{M}})=2 M$. Furthermore, by inspection, a possible basis for the right nullspace of $\breve{M}$ is given by

$$
\mathcal{N}(\breve{\mathbf{M}})=\underset{\text { col. }}{\operatorname{span}}\left[\begin{array}{cc}
\mathbf{I}_{2} & \mathbf{J p}_{R_{k_{o}}} \\
\mathbf{0}_{1 \times 2} & 1 \\
\mathbf{I}_{2} & \mathbf{J p}_{L_{1}} \\
\vdots & \vdots \\
\mathbf{I}_{2} & \mathbf{J p}_{L_{M}}
\end{array}\right]
$$


Note the similarity of this result with that of (18). Clearly, the physical interpretation of this result is analogous to that of the single-landmark case: the global translation and orientation of the state vector are unobservable.

2) Standard EKF-SLAM: We now study the observability properties of the standard EKF-SLAM, in which the Jacobians are evaluated at the latest state estimates (i.e., $\mathbf{x}_{k \mid k-1}^{\star}=\hat{\mathbf{x}}_{k \mid k-1}$ and $\mathbf{x}_{k \mid k}^{\star}=\hat{\mathbf{x}}_{k \mid k}$, for all $k$ ). Once again, we begin by examining the single-landmark case. By deriving an expression analogous to that of (26), we obtain (cf. Section IV-B1):

$\boldsymbol{\Phi}_{R_{k_{o}+1}} \boldsymbol{\Phi}_{R_{k_{o}}}=\left[\begin{array}{cc}\mathbf{I}_{2} & \mathbf{J}\left(\hat{\mathbf{p}}_{R_{k_{o}+2 \mid k_{o}+1}}-\hat{\mathbf{p}}_{R_{k_{o} \mid k_{o}}}-\Delta \mathbf{p}_{R_{k_{o}+1}}\right) \\ \mathbf{0}_{1 \times 2} & 1\end{array}\right]$

where $\Delta \mathbf{p}_{R_{k_{o}+1}} \triangleq \hat{\mathbf{p}}_{R_{k_{o}+1 \mid k_{o}+1}}-\hat{\mathbf{p}}_{R_{k_{o}+1 \mid k_{o}}}$ is the correction in the robot position due to the EKF update at time-step $k_{o}+1$. Using induction, we can show that:

$$
\begin{aligned}
& \boldsymbol{\Phi}_{R_{k_{o}+\ell-1}} \boldsymbol{\Phi}_{R_{k_{o}+\ell-2}} \cdots \boldsymbol{\Phi}_{R_{k_{o}}}= \\
& {\left[\begin{array}{cc}
\mathbf{I}_{2} & \mathbf{J}\left(\hat{\mathbf{p}}_{R_{k_{o}+\ell \mid k_{o}+\ell-1}}-\hat{\mathbf{p}}_{R_{k_{o} \mid k_{o}}}-\sum_{j=k_{o}+1}^{k_{o}+\ell-1} \Delta \mathbf{p}_{R_{j}}\right) \\
\mathbf{0}_{1 \times 2} & 1
\end{array}\right]}
\end{aligned}
$$

where $\ell>0$. Therefore (cf. (11), (12), and (13))

$$
\begin{aligned}
& \mathbf{H}_{R_{k_{o}+\ell}} \boldsymbol{\Phi}_{R_{k_{o}+\ell-1}} \cdots \boldsymbol{\Phi}_{R_{k_{o}}}=\mathbf{H}_{L_{k_{o}+\ell}}
\end{aligned}
$$

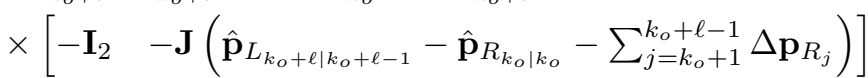

Using this result, we can write $\mathbf{M}$ (cf. (24)) as:

$$
\begin{aligned}
& \mathbf{M}=\underbrace{\operatorname{Diag}\left(\mathbf{H}_{L_{k_{o}}}, \mathbf{H}_{L_{k_{o}+1}}, \cdots, \mathbf{H}_{L_{k_{o}+m}}\right)}_{\mathbf{D}}
\end{aligned}
$$

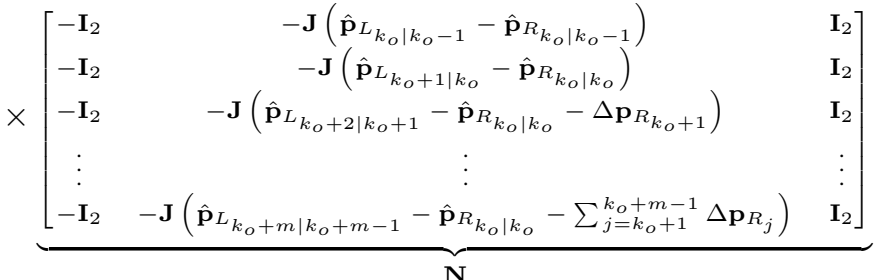

Lemma 4.4: The rank of the observability matrix, $\mathbf{M}$, of the system model of the standard EKF is equal to 3 .

Proof: First, we note that the estimates of any given state variable at different time instants are generally different. Hence, in contrast to the case of the ideal EKF-SLAM, the following inequalities generally hold: $\hat{\mathbf{p}}_{R_{k_{o}+i \mid k_{o}+i-1}} \neq$ $\hat{\mathbf{p}}_{R_{k_{O}+i \mid k_{O}+i}}$ and $\hat{\mathbf{p}}_{L_{k_{O}+i \mid k_{O}+i-1}} \neq \hat{\mathbf{p}}_{L_{k_{O}+\ell \mid k_{O}+\ell-1}}$, for $i \neq \ell$. Therefore, the third column of $\mathbf{N}$ will be, in general, a vector with unequal elements, and thus $\operatorname{rank}(\mathbf{N})=3$. Proceeding similarly to the proof of Lemma 4.3 , we first find one possible basis for the range space of $\mathbf{N}, \mathcal{R}(\mathbf{N})$. By inspection, we see that such a basis is given simply by the first 3 columns of $\mathbf{N}$, which we denote by $\mathbf{u}_{i}(i=1,2,3)$. Moreover, it can be verified that generally $\mathrm{Du}_{i} \neq \mathbf{0}$. Therefore, $\operatorname{dim}(\mathcal{N}(\mathbf{D}) \bigcap \mathcal{R}(\mathbf{N}))=0$, and finally $\operatorname{rank}(\mathbf{M})=\operatorname{rank}(\mathbf{N})-$ $\operatorname{dim}(\mathcal{N}(\mathbf{D}) \bigcap \mathcal{R}(\mathbf{N}))=\operatorname{rank}(\mathbf{N})=3$.

We thus see that the linearized error-state model employed in the standard EKF-SLAM has different observability properties than that of the ideal EKF-SLAM (cf. Lemma 4.3) and that of the underlying nonlinear system (cf. Lemma 4.2). In particular, by processing the measurements collected in the interval $\left[k_{o}, k_{o}+m\right]$, the filter acquires information in 3 dimensions of the state space (along the directions corresponding to the observable subspace of the EKF). However, the measurements actually provide information in only 2 directions of the state space (i.e., the robot-to-landmark relative position). As a result, the EKF gains "spurious information" along the unobservable directions of the underlying nonlinear SLAM system, which leads to inconsistency.

To probe further, we note that the basis of the right nullspace of $\mathbf{M}$ is given by:

$$
\mathcal{N}(\mathbf{M})=\underset{\text { col. }}{\operatorname{span}}\left[\begin{array}{c}
\mathbf{I}_{2} \\
\mathbf{0}_{1 \times 2} \\
\mathbf{I}_{2}
\end{array}\right]=\operatorname{span}\left[\begin{array}{ll}
\mathbf{n}_{1} & \mathbf{n}_{2}
\end{array}\right]
$$

Note that these two vectors correspond to a shifting of the $x-y$ plane, which implies that such a shifting is unobservable. On the other hand, the direction corresponding to the global orientation is "missing" from the unobservable subspace of the EKF system model (cf. (18) and (19)). Therefore, we see that the filter will gain "nonexistent" information about the robot's global orientation. This will lead to an unjustified reduction in the orientation uncertainty, which will, in turn, further reduce the uncertainty in all the state variables. This agrees in some respects with [Bailey et al., 2006], [Huang and Dissanayake, 2007], where it was argued that the orientation uncertainty is the main cause of the filter's inconsistency in SLAM. However, we point out that the root cause of the problem is that the linearization points used for computing the Jacobians in the standard EKF-SLAM (i.e., the latest state estimates) change the dimension of the observable subspace, and thus fundamentally alter the properties of the estimation process.

Identical conclusions can be drawn when $M>1$ landmarks are included in the state vector (cf. [Huang et al., 2008c]). For this general case, the nullspace of the observability matrix can be shown to be equal to:

$$
\mathcal{N}(\mathbf{M})=\operatorname{span}\left[\begin{array}{c}
\mathbf{I}_{2} \\
\mathbf{0}_{1 \times 2} \\
\mathbf{I}_{2} \\
\vdots \\
\mathbf{I}_{2}
\end{array}\right]
$$

We thus see that the global orientation is erroneously observable in this case as well, which leads to inconsistent estimates.

An interesting remark is that the covariance matrices of the system and measurement noise do not appear in the observability analysis of the filter's system model. Therefore, even if these covariance matrices are artificially inflated, the filter will retain the same observability properties (i.e., the same observable and unobservable subspaces). This shows that no amount of covariance inflation can result in correct observability properties. Similarly, even if the iterated EKF [BarShalom et al., 2001] is employed for state estimation, the same, erroneous, observability properties will arise, since the landmark position estimates will generally differ at different time steps. 


\section{ObServability-CONSTRAined EKF Design}

In the preceding section, it was shown that when the EKF Jacobians are evaluated using the latest state estimates, the EKF error-state model has an observable subspace of dimension higher than the actual nonlinear SLAM system. This will always lead to unjustified reduction of the covariance estimates, and thus inconsistency. We now describe a framework for addressing this problem.

Our key conjecture is that, by ensuring an unobservable subspace of appropriate dimension, we can avoid the influx of spurious information in the erroneously observable direction of the state space, and thus improve the consistency of the estimates. Therefore, we propose selecting the linearization points of the EKF in a way that guarantees an unobservable subspace of dimension 3 for the linearized error-state model. This corresponds to satisfying conditions (40)-(41) of the following lemma:

Lemma 5.1: If the linearization points, $\mathbf{x}_{k \mid k}^{\star}$ and $\mathbf{x}_{k+1 \mid k}^{\star}$, at which the EKF Jacobians $\boldsymbol{\Phi}_{k}=\boldsymbol{\Phi}_{k}\left(\mathbf{x}_{R_{k+1 \mid k}}^{\star}, \mathbf{x}_{R_{k \mid k}}^{\star}\right)$ and $\mathbf{H}_{k+1}=\mathbf{H}_{k+1}\left(\mathbf{x}_{R_{k+1 \mid k}}^{\star}, \mathbf{p}_{L_{k+1 \mid k}}^{\star}\right)$ are evaluated, are selected so as to fulfill the conditions:

$$
\begin{aligned}
& \mathbf{H}_{k_{o}} \mathbf{U}=\mathbf{0}, \text { for } \ell=0 \\
& \mathbf{H}_{k_{o}+\ell} \boldsymbol{\Phi}_{k_{o}+\ell-1} \cdots \boldsymbol{\Phi}_{k_{o}} \mathbf{U}=\mathbf{0}, \quad \forall \ell>0
\end{aligned}
$$

where $\mathbf{U}$ is a $5 \times 3$ full-rank matrix, then the corresponding observability matrix is of rank 2 .

Proof: When (40)-(41) hold, then all the block rows of the observability matrix (cf. (23)) will have the same nullspace, spanned by the columns of $\mathbf{U}$.

Essentially, the selection of $\mathbf{U}$ is a design choice, which allows us to control the unobservable subspace of the EKF system model. Ideally we would like the column vectors of $\mathbf{U}$ to be identical to those in (18), which define the unobservable directions of the actual, nonlinear SLAM system. However, this cannot be achieved in practice, since these directions depend on the true values of the state, which are unavailable during any real-world implementation.

A natural selection, which is realizable in practice, is to define the unobservable subspace of the observability matrix based on the state estimates at the first time instant a landmark was detected, i.e., for the single-landmark case to choose ${ }^{3}$

$$
\mathbf{U}=\left[\begin{array}{cc}
\mathbf{I}_{2} & \mathbf{J} \hat{\mathbf{p}}_{R_{k_{o} \mid k_{o}-1}} \\
\mathbf{0}_{1 \times 2} & 1 \\
\mathbf{I}_{2} & \mathbf{J} \hat{\mathbf{p}}_{L_{k_{o} \mid k_{o}}}
\end{array}\right]
$$

which satisfies condition (40).

We stress that this is just one of several approaches for selecting the matrix U. For instance, one limitation with this approach is that, in cases where the initial estimates of the landmarks are not of sufficient accuracy, the subspace defined in this manner might not be close to the actual unobservable subspace. To address this problem one can employ advanced techniques for landmark initialization (e.g., delayed-state initialization [Leonard et al., 2002]), to obtain more precise initial

\footnotetext{
${ }^{3}$ When multiple $(M>1)$ landmarks are included in the state vector, $\mathbf{U}$ can be chosen analogously, augmented by a new block row, $\left[\begin{array}{ll}\mathbf{I}_{2} & \mathbf{J}_{\mathbf{p}_{i}, k_{o} \mid k_{o}}\end{array}\right]$, corresponding to each landmark, $L_{i}(i=1,2, \ldots, M)$ [Huang et al., 2008c].
}

estimates, and use these to define a matrix $\mathbf{U}$. This approach, which could lead to improved accuracy in certain situations, is one of several interesting options to explore within the proposed design methodology.

Once $\mathbf{U}$ has been selected, the next design decision to be made is the choice of the linearization points at each time step. For the particular selection of $\mathbf{U}$ in (42), this amounts to choosing the linearization points for all $k>k_{o}$ to ensure that (41) holds (note that (40) is satisfied by construction in this case). Clearly, several options exist, each of which leads to a different algorithm within the general framework described here. In what follows, we present two approaches to achieve this goal.

\section{A. First Estimates Jacobian (FEJ)-EKF}

We first describe the First Estimates Jacobian (FEJ)EKF estimator that was originally proposed in our previous work [Huang et al., 2008a], [Huang et al., 2008b]. The key idea of this approach is to choose the first-ever available estimates for all the state variables as the linearization points. In particular, compared to the standard EKF, the following two changes are required in the way that the Jacobians are evaluated:

1) Instead of computing the state-propagation Jacobian matrix $\Phi_{R_{k}}$ as in (8), we employ the expression:

$$
\boldsymbol{\Phi}_{R_{k}}^{\prime}=\left[\begin{array}{cc}
\mathbf{I}_{2} & \mathbf{J}\left(\hat{\mathbf{p}}_{R_{k+1 \mid k}}-\hat{\mathbf{p}}_{R_{k \mid k-1}}\right) \\
\mathbf{0}_{1 \times 2} & 1
\end{array}\right]
$$

The difference compared to (8) is that the prior robot position estimate, $\hat{\mathbf{p}}_{R_{k \mid k-1}}$, is used in place of the posterior estimate, $\hat{\mathbf{p}}_{R_{k \mid k}}$.

2) In the evaluation of the measurement Jacobian matrix $\mathbf{H}_{k+1}$ (cf. (11), (12), and (13)), we always utilize the landmark estimate from the first time the landmark was detected and initialized. Thus, if a landmark was first seen at time-step $k_{o}$, we compute the measurement Jacobian as:

$$
\begin{aligned}
& \mathbf{H}_{k+1}^{\prime}=\left[\begin{array}{ll}
\mathbf{H}_{R_{k+1}}^{\prime} & \mathbf{H}_{L_{k+1}}^{\prime}
\end{array}\right] \\
& =\left(\nabla \mathbf{h}_{k+1}\right) \mathbf{C}^{T}\left(\hat{\phi}_{R_{k+1 \mid k}}\right)
\end{aligned}
$$

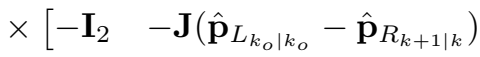

As a result of the above modifications, only the first estimates of all landmark positions and all robot poses appear in the filter Jacobians. It is easy to verify that the above Jacobians satisfy (40) and (41) for the choice of $\mathbf{U}$ in (42). Thus, the FEJ-EKF is based on an error-state system model whose unobservable subspace is of dimension 3.

\section{B. Observability Constrained (OC)-EKF}

Even though the FEJ-EKF typically performs substantially better than the standard EKF (cf. Sections VI and VII), it relies heavily on the initial state estimates, since it uses them at all time steps for computing the filter Jacobians. If these estimates are far from the true state, the linearization errors incurred may be large, and could degrade the performance of the estimator. As a motivating example, consider the linearization 
of a general, scalar nonlinear function $f(x)$ around a point $x^{\star}$. By employing Taylor expansion, we obtain:

$$
f(x)=f\left(x^{\star}\right)+f^{\prime}\left(x^{\star}\right)\left(x-x^{\star}\right)+\frac{f^{\prime \prime}(\xi)}{2}\left(x-x^{\star}\right)^{2}
$$

In this expression, which holds in the interval $\left(x, x^{\star}\right), f^{\prime}$ and $f^{\prime \prime}$ are the first- and second-order derivatives of $f$, and $\xi \in\left(x, x^{\star}\right)$. The last term in the above expression, $\frac{f^{\prime \prime}(\xi)}{2}\left(x-x^{\star}\right)^{2}$, describes the linearization error, which should be kept as small as possible to maintain the validity of the linear approximation. Since we do not have control over the term $f^{\prime \prime}(\xi)$, to keep the linearization error small, we see that the term $\left(x-x^{\star}\right)^{2}$ should be kept as small as possible.

An interesting observation is that if $x$ in the above example is a Gaussian random variable with mean $\hat{x}$, then the expected value of $\left(x-x^{\star}\right)^{2}$ is minimized by choosing $x^{\star}=\hat{x}$. This is precisely what the standard EKF does: at each time step, it employs the mean of the state for computing the linearization Jacobians. This leads to small linearization error for each time step, but as explained in Section IV-B2, it also changes the observability properties of the SLAM system model, and adversely affects performance.

The above discussion shows that, in the context of SLAM, there are two competing goals that should be reconciled: reduced linearization errors at each time step and correct observability properties of the linearized system model. Therefore, we propose selecting the linearization points of the EKF so as to minimize the expected squared error of the linearization points while satisfying the observability conditions (40)-(41). This can be formulated as a constrained minimization problem where the constraints express the observability requirements. Thus we term the resulting filter Observability-Constrained (OC)-EKF.

Specifically, at time-step $k+1$, we aim at minimizing the linearization error of the points $\mathbf{x}_{R_{k \mid k}}^{\star}$ and $\mathbf{x}_{k+1 \mid k}^{\star}$, which appear in the Jacobians $\boldsymbol{\Phi}_{k}$ and $\mathbf{H}_{k+1}$, subject to the observability constraint (41). Mathematically, this is expressed as:

$$
\begin{array}{cc}
\min _{\mathbf{x}_{R_{k \mid k}}^{\star}, \mathbf{x}_{k+1 \mid k}^{\star}} & \left(\int\left\|\mathbf{x}_{R_{k}}-\mathbf{x}_{R_{k \mid k}}^{\star}\right\|^{2} p\left(\mathbf{x}_{R_{k}} \mid \mathbf{z}_{0: k}\right) d \mathbf{x}_{R_{k}}+\right. \\
& \left.\int\left\|\mathbf{x}_{k+1}-\mathbf{x}_{k+1 \mid k}^{\star}\right\|^{2} p\left(\mathbf{x}_{k+1} \mid \mathbf{z}_{0: k}\right) d \mathbf{x}_{k+1}\right) \\
\text { s.t. } & \mathbf{H}_{k+1} \mathbf{\Phi}_{k} \cdots \mathbf{\Phi}_{k_{o}} \mathbf{U}=\mathbf{0}, \quad \forall k \geq k_{o}
\end{array}
$$

where $\mathbf{z}_{0: k}$ denotes all the measurements available during the time interval $[0, k]$. Note that only the robot pose appears in the Jacobians of the propagation model (cf. (6)), while both the robot pose and the landmark positions appear in the Jacobians of the measurement equations (cf. (11)). This justifies the choice of the above cost function.

In general, the constrained minimization problem (46)(47) is intractable. However, when the two pdfs, $p\left(\mathbf{x}_{R_{k}} \mid \mathbf{z}_{0: k}\right)$ and $p\left(\mathbf{x}_{k+1} \mid \mathbf{z}_{0: k}\right)$, are Gaussian distributions (which is the assumption employed in the EKF), we can solve the problem analytically and find a closed-form solution. In the following, we show how the closed-form solution can be computed for the simple case where only one landmark is included in the state vector. The case of multiple landmarks is presented in [Huang et al., 2008c].
We note that the following lemma will be helpful for the ensuing derivations:

Lemma 5.2: The constrained optimization problem (46)(47) is equivalent to the following:

$$
\begin{aligned}
& \min _{\mathbf{x}_{R_{k \mid k}}^{\star}, \mathbf{x}_{k+1 \mid k}^{\star}}\left\|\hat{\mathbf{x}}_{R_{k \mid k}}-\mathbf{x}_{R_{k \mid k}}^{\star}\right\|^{2}+\left\|\hat{\mathbf{x}}_{k+1 \mid k}-\mathbf{x}_{k+1 \mid k}^{\star}\right\|^{2} \\
& \text { s.t. } \quad \mathbf{p}_{L_{k+1 \mid k}}^{\star}-\mathbf{p}_{R_{k \mid k}}^{\star}=\hat{\mathbf{p}}_{L_{k_{o} \mid k_{o}}}-\mathbf{p}_{R_{k \mid k-1}}^{\star}+\sum_{j=k_{o}}^{k-1} \Delta \mathbf{p}_{R_{j}}^{\star}
\end{aligned}
$$

where $\Delta \mathbf{p}_{R_{j}}^{\star} \triangleq \mathbf{p}_{R_{j \mid j}}^{\star}-\mathbf{p}_{R_{j \mid j-1}}^{\star}$

Proof: See Appendix D.

Using the technique of Lagrangian multipliers, the optimal solution to the problem (48)-(49) can be obtained as:

$$
\begin{aligned}
\mathbf{p}_{R_{k \mid k}}^{\star} & =\hat{\mathbf{p}}_{R_{k \mid k}}+\frac{\boldsymbol{\lambda}_{k}}{2}, & \phi_{R_{k \mid k}}^{\star} & =\hat{\phi}_{R_{k \mid k}}, \\
\mathbf{x}_{R_{k+1 \mid k}}^{\star} & =\hat{\mathbf{x}}_{R_{k+1 \mid k}}, & \mathbf{p}_{L_{k+1 \mid k}}^{\star} & =\hat{\mathbf{p}}_{L_{k+1 \mid k}}-\frac{\boldsymbol{\lambda}_{k}}{2}
\end{aligned}
$$

with

$\boldsymbol{\lambda}_{k}=\left(\hat{\mathbf{p}}_{L_{k+1 \mid k}}-\hat{\mathbf{p}}_{L_{k_{o} \mid k_{o}}}\right)-\left(\hat{\mathbf{p}}_{R_{k \mid k}}-\mathbf{p}_{R_{k \mid k-1}}^{\star}+\sum_{j=k_{o}}^{k-1} \Delta \mathbf{p}_{R_{j}}^{\star}\right)$

Note that in the case where multiple landmarks are included in the state vector, each landmark imposes a constraint analogous to (49), and thus the analytical solution of the optimal linearization points can be obtained similarly [Huang et al., 2008c].

Using the linearization points in (50), the filter Jacobians in the OC-EKF are now computed as follows:

1) The state-propagation Jacobian matrix is calculated as:

$$
\mathbf{\Phi}_{R_{k}}^{\prime \prime}=\left[\begin{array}{cc}
\mathbf{I}_{2} & \mathbf{J}\left(\hat{\mathbf{p}}_{R_{k+1 \mid k}}-\hat{\mathbf{p}}_{R_{k \mid k}}-\frac{\boldsymbol{\lambda}_{k}}{2}\right) \\
\mathbf{0}_{1 \times 2} & 1
\end{array}\right]
$$

2) The measurement Jacobian matrix is calculated as:

$$
\begin{aligned}
& \mathbf{H}_{k+1}^{\prime \prime}=\left[\begin{array}{ll}
\mathbf{H}_{R_{k+1}}^{\prime \prime} & \mathbf{H}_{L_{k+1}}^{\prime \prime}
\end{array}\right] \\
& =\left(\nabla \mathbf{h}_{k+1}\right) \mathbf{C}^{T}\left(\hat{\phi}_{R_{k+1 \mid k}}\right)
\end{aligned}
$$

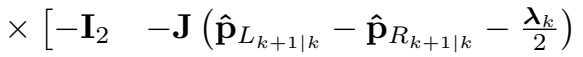

It is important to note that, compared to the FEJ-EKF, the OC-EKF not only guarantees the correct observability properties of the EKF linearized system model (so does the FEJ-EKF), but also minimizes the linearization errors under the given observability requirements. The simulation and experimental results presented in Sections VI and VII show the OC-EKF attains slightly better performance than the FEJ-EKF. We also point out that, compared to the standard EKF, the only change in the OC-EKF is the way in which the Jacobians are computed. The state estimates in the OC-EKF are propagated and updated in the same way as in the standard EKF, as outlined in Algorithm 1. In addition, we stress that both the FEJ-EKF and OC-EKF estimators are also causal and realizable "in the real world," since they do not utilize any knowledge of the true state. Interestingly, although both the FEJ-EKF and the OC-EKF do not use the latest available state estimates (and thus utilize Jacobians that are less accurate 
than those of the standard EKF), both simulation tests and realworld experiments demonstrate that they perform significantly better than the standard EKF in terms of consistency and accuracy (cf. Sections VI and VII).

Algorithm 1 Observability Constrained (OC)-EKF SLAM

Propagation: When an odometry measurement is received:

- propagate the robot pose estimate, via (2)-(3)

- compute the robot pose propagation Jacobian (cf. (51))

- propagate the state covariance matrix:

$$
\mathbf{P}_{k+1 \mid k}=\boldsymbol{\Phi}_{k}^{\prime \prime} \mathbf{P}_{k \mid k} \boldsymbol{\Phi}_{k}^{\prime \prime T}+\mathbf{G}_{k} \mathbf{Q}_{k} \mathbf{G}_{k}^{T}
$$

where

$$
\boldsymbol{\Phi}_{k}^{\prime \prime}=\operatorname{Diag}\left(\boldsymbol{\Phi}_{R_{k}}^{\prime \prime}, \mathbf{I}_{2 M}\right) \text { and } \mathbf{G}_{k}=\left[\begin{array}{ll}
\mathbf{G}_{R_{k}}^{T} & \mathbf{0}_{(2 M) \times 2}^{T}
\end{array}\right]^{T}
$$

Update: When a robot-to-landmark measurement is received:

- compute the measurement residual:

$$
\mathbf{r}_{k+1}=\mathbf{z}_{k+1}-\mathbf{h}\left(\hat{\mathbf{x}}_{k+1 \mid k}\right)
$$

- compute the measurement Jacobian matrix (cf. (52))

- compute the Kalman gain:

$$
\mathbf{K}_{k+1}=\mathbf{P}_{k+1 \mid k} \mathbf{H}_{k+1}^{\prime^{T}} \mathbf{S}_{k+1}^{-1}
$$

with

$$
\mathbf{S}_{k+1}=\mathbf{H}_{k+1}^{\prime \prime} \mathbf{P}_{k+1 \mid k} \mathbf{H}_{k+1}^{\prime \prime^{T}}+\mathbf{R}_{k+1}
$$

- update the state estimate:

$$
\hat{\mathbf{x}}_{k+1 \mid k+1}=\hat{\mathbf{x}}_{k+1 \mid k}+\mathbf{K}_{k+1} \mathbf{r}_{k+1}
$$

- update the state covariance matrix:

$$
\mathbf{P}_{k+1 \mid k+1}=\mathbf{P}_{k+1 \mid k}-\mathbf{K}_{k+1} \mathbf{S}_{k+1} \mathbf{K}_{k+1}^{T}
$$

\section{Relation to Prior Work}

At this point, it is interesting to examine the relation of our analysis, which addresses the general case of a moving robot, to the previous work that has focused on special cases [Julier and Uhlmann, 2001], [Huang and Dissanayake, 2007]. We first note that the "correct" observability properties of the FEJ-EKF and OC-EKF are attributed to the fact that conditions (40)-(41) hold, which is not the case for the standard EKF. Thus, (40)(41) can be seen as sufficient conditions that, when satisfied by the filter Jacobians, ensure that the observability matrix has a nullspace of appropriate dimensions. Note also that, due to the identity (27), the conditions (40)-(41) are trivially satisfied by the ideal EKF with null space $\mathbf{U}=\left[\begin{array}{lll}\mathbf{n}_{1} & \mathbf{n}_{2} & \mathbf{n}_{3}\end{array}\right]$ (cf. (18)). In what follows, we show that the conditions (40)(41) encompass the ones derived in [Julier and Uhlmann, 2001] and [Huang and Dissanayake, 2007] as special cases.

1) Stationary robot: We first examine the special case studied in [Julier and Uhlmann, 2001], where the robot remains stationary, while observing the relative position of a single landmark. In [Julier and Uhlmann, 2001] the following Jacobian constraint for consistent estimation was derived (cf.
Theorem 1 therein):

$$
\begin{aligned}
& \nabla \mathbf{h}^{\mathbf{x}}-\nabla \mathbf{h}^{\mathbf{p}} \nabla \mathbf{g}^{\mathbf{x}}=\mathbf{0} \\
& \Leftrightarrow \mathbf{H}_{R_{k}}+\mathbf{H}_{L_{k}} \nabla \mathbf{g}^{\mathbf{x}}=\mathbf{0} \\
& \Leftrightarrow\left[\begin{array}{ll}
\mathbf{H}_{R_{k}} & \mathbf{H}_{L_{k}}
\end{array}\right]\left[\begin{array}{c}
\mathbf{I}_{3} \\
\nabla \mathbf{g}^{\mathbf{x}}
\end{array}\right]=\mathbf{0} \\
& \Leftrightarrow \mathbf{H}_{k} \mathbf{U}_{s}=\mathbf{0}
\end{aligned}
$$

where, using our notation, $\nabla \mathbf{h}^{\mathbf{x}}=\mathbf{H}_{R_{k}}$ and $-\nabla \mathbf{h}^{\mathbf{p}}=\mathbf{H}_{L_{k}}$ are the measurement Jacobian matrices with respect to the robot pose and landmark position, respectively, and $\nabla \mathbf{g}^{\mathbf{x}}$ is the landmark initialization Jacobian with respect to the robot pose at time-step $k_{o}$. Note that the condition (53) is identical to the one in (40) for the special case of a stationary robot.

Remarkably, the space spanned by the columns of the matrix $\mathbf{U}_{s}$, for this special case, is same as the one spanned by the columns of $\mathbf{U}$ in (42). To see that, we first need to derive an expression for $\nabla \mathbf{g}^{\mathbf{x}}$. In [Julier and Uhlmann, 2001], a relative-position measurement model is employed (by combining a distance and a bearing measurement), and thus the initialization function $\mathbf{g}(\cdot)$ is given by:

$$
\mathbf{p}_{L_{k_{o}}}=\mathbf{g}\left(\mathbf{x}_{R_{k_{o}}}, \mathbf{z}_{k_{o}}, \mathbf{v}_{k_{o}}\right)=\mathbf{C}\left(\phi_{R_{k_{o}}}\right)\left(\mathbf{z}_{k_{o}}-\mathbf{v}_{k_{o}}\right)+\mathbf{p}_{R_{k_{o}}}
$$

where $\mathbf{z}_{k_{o}}$ is the first measurement of the landmark's relative position and $\mathbf{v}_{k_{o}}$ denotes the noise in this measurement. Evaluating the derivative of this function with respect to the robot pose at the current state estimate we have:

$$
\begin{aligned}
\nabla \mathbf{g}^{\mathbf{x}} & =\left[\begin{array}{ll}
\mathbf{I}_{2} & \mathbf{J C}\left(\hat{\phi}_{R_{k_{o} \mid k_{o}-1}}\right) \mathbf{z}_{k_{o}}
\end{array}\right] \\
& =\left[\begin{array}{ll}
\mathbf{I}_{2} & \mathbf{J}\left(\hat{\mathbf{p}}_{L_{k_{o} \mid k_{o}}}-\hat{\mathbf{p}}_{R_{k_{o} \mid k_{o}-1}}\right)
\end{array}\right]
\end{aligned}
$$

where this last equation results from taking conditional expectations on both sides of (54) and solving for $\mathbf{z}_{k_{\circ}}$.

Substituting (55) in the expression for $\mathbf{U}_{s}$ (cf. (53)), yields:

$$
\mathbf{U}_{s}=\left[\begin{array}{cc}
\mathbf{I}_{2} & \mathbf{0}_{2 \times 1} \\
\mathbf{0}_{1 \times 2} & 1 \\
\mathbf{I}_{2} & \mathbf{J}\left(\hat{\mathbf{p}}_{L_{k_{o} \mid k_{o}}}-\hat{\mathbf{p}}_{R_{k_{o} \mid k_{o}-1}}\right)
\end{array}\right]
$$

One can easily verify that $\mathbf{U}_{s}$ and $\mathbf{U}$ span the same column space by noting that $\mathbf{U}_{s}\left[\begin{array}{cc}\mathbf{I}_{2} & \mathbf{J} \hat{\mathbf{p}}_{R_{k_{o} \mid k_{o}-1}} \\ \mathbf{0}_{1 \times 2} & 1\end{array}\right]=\mathbf{U}$.

2) Moving robot with one-step motion: We now consider the special case studied in [Huang and Dissanayake, 2007], where a robot observes a landmark, moves once and then reobserves the landmark. In [Huang and Dissanayake, 2007], the key Jacobian relationship that needs to be satisfied in order to obtain consistent estimation in this case (cf. Theorem 4.2 therein) is given by:

$$
A_{e}=B_{e} \nabla f_{\phi X_{r}}^{A}
$$

Using our notation, the above matrices are written as:

$$
\begin{aligned}
\nabla f_{\phi X_{r}}^{A} & =\boldsymbol{\Phi}_{R_{k_{o}}} \\
A_{e} & =-\mathbf{H}_{L_{k_{o}}}^{-1} \mathbf{H}_{R_{k_{o}}} \\
B_{e} & =-\mathbf{H}_{L_{k_{o}+1}}^{-1} \mathbf{H}_{R_{k_{o}+1}}
\end{aligned}
$$


Substituting in (56) and rearranging terms yields:

$$
\begin{array}{r}
\mathbf{H}_{L_{k_{o}+1}}^{-1} \mathbf{H}_{R_{k_{o}+1}} \mathbf{\Phi}_{R_{k_{o}}}-\mathbf{H}_{L_{k_{o}}}^{-1} \mathbf{H}_{R_{k_{o}}}=\mathbf{0} \\
\Leftrightarrow\left[\begin{array}{ll}
\mathbf{H}_{R_{k_{o}+1}} & \mathbf{H}_{L_{k_{o}+1}}
\end{array}\right]\left[\begin{array}{cc}
\mathbf{\Phi}_{R_{k_{o}}} & \mathbf{0}_{3 \times 2} \\
\mathbf{0}_{3 \times 2}^{T} & \mathbf{I}_{2}
\end{array}\right]\left[\begin{array}{c}
\mathbf{I}_{3} \\
-\mathbf{H}_{L_{k_{o}}}^{-1} \mathbf{H}_{R_{k_{o}}}
\end{array}\right]=\mathbf{0} \\
\Leftrightarrow \mathbf{H}_{k_{o}+1} \mathbf{\Phi}_{k_{o}} \mathbf{U}_{1}=\mathbf{0}
\end{array}
$$

which is the same as the condition in (41) for the special case of $\ell=1$ (i.e., the robot moves only once). Additionally, it is easy to verify that $\mathbf{H}_{k_{o}} \mathbf{U}_{1}=\mathbf{0}$, which corresponds to condition (40). Moreover, it is fairly straightforward to show that for the case of distance and bearing measurements considered in [Huang and Dissanayake, 2007], the matrix $\mathbf{U}_{1}$ spans the same column space as $\mathbf{U}$ in (42). This analysis demonstrates that the Jacobian constraints (40)-(41) derived based on the observability criterion are general, and encompass the condition of [Huang and Dissanayake, 2007] as a special case.

\section{Simulation Results}

A series of Monte-Carlo comparison studies were conducted under various conditions, in order to validate the preceding theoretical analysis and to demonstrate the capability of the FEJ-EKF and OC-EKF estimators to improve the consistency of EKF-SLAM. The metrics used to evaluate filter performance are: (i) the RMS error, and (ii) the average normalized (state) estimation error squared (NEES) [Bar-Shalom et al., 2001]. Specifically, for the landmarks we compute the average RMS errors and average NEES by averaging the squared errors and the NEES, respectively, over all Monte Carlo runs, all landmarks, and all time steps. On the other hand, for the robot pose we compute these error metrics by averaging over all Monte Carlo runs for each time step (cf. [Huang et al., 2008c] for a more detailed description).

The RMS of the estimation errors provides us with a concise metric of the accuracy of a given estimator. On the other hand, the NEES is a metric for evaluating filter consistency. Specifically, it is known that the NEES of an $N$-dimensional Gaussian random variable follows a $\chi^{2}$ distribution with $N$ degrees of freedom. Therefore, if a certain filter is consistent, we expect that the average NEES for the robot pose will be close to 3 for all $k$, and that the average landmark NEES will be close to 2. The larger the deviations of the NEES from these values, the worse the inconsistency of the filter. By studying both the RMS errors and NEES of all the filters considered here, we obtain a comprehensive picture of the estimators' performance.

In the simulation tests presented in this section, two SLAM scenarios with loop closure were considered. In the first case, a robot moves on a circular trajectory and continuously observes 2 landmarks, while in the second case the robot sequentially observes 20 landmarks in total.

\section{A. First Simulation: Always Observing 2 Landmarks}

To validate the preceding observability analysis, we first ran a SLAM simulation where a robot executes 80 loops on a circular trajectory, and continuously observes 2 landmarks at

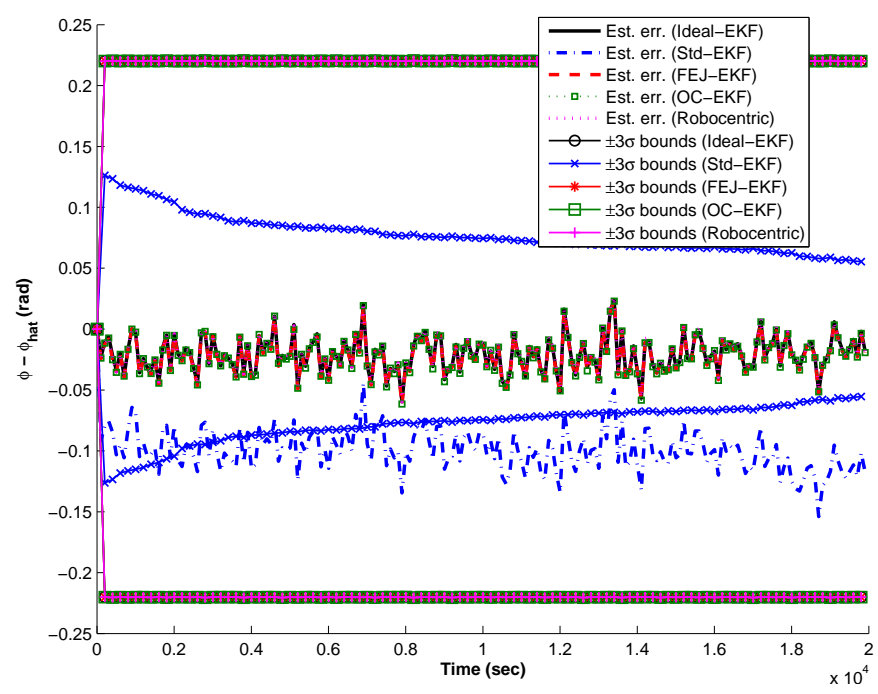

Fig. 1. Orientation estimation errors vs. $3 \sigma$ bounds obtained from one typical realization of the Monte Carlo simulations. The $\sigma$ values are computed as the square-root of the corresponding diagonal element of the estimated covariance matrix. Note that the estimation errors and the $3 \sigma$ bounds of the ideal EKF, the FEJ-EKF, the OC-EKF and the robocentric mapping filter are almost identical, which makes the corresponding lines difficult to distinguish.

every time step. Note that this simulation was run sufficiently long to ensure that the filters (approximately) reach their steady states and thus exhibit divergence (if any) more clearly. In this simulation, all filters process the same data, to ensure a fair comparison. The five EKF estimators compared are: (1) the ideal EKF, (2) the standard EKF, (3) the FEJ-EKF, (4) the OC-EKF, and (5) the robocentric mapping filter presented in [Castellanos et al., 2004], which aims at improving the consistency of SLAM by expressing the landmarks in a robotrelative frame.

For the results presented in this section, a robot with a simple differential drive model moves on a planar surface, at a constant linear velocity of $v=0.25 \mathrm{~m} / \mathrm{sec}$. The two drive wheels are equipped with encoders that measure revolutions and provide measurements of velocity (i.e., right and left wheel velocities, $v_{r}$ and $v_{l}$, respectively) with standard deviation equal to $\sigma=5 \% v$ for each wheel. These measurements are used to obtain linear and rotational velocity measurements for the robot, which are given by $v=\frac{v_{r}+v_{l}}{2}$ and $\omega=\frac{v_{r}-v_{l}}{a}$, where $a=0.5 \mathrm{~m}$ is the distance between the drive wheels. Thus, the standard deviations of the linear and rotational velocity measurements are $\sigma_{v}=\frac{\sqrt{2}}{2} \sigma$ and $\sigma_{\omega}=\frac{\sqrt{2}}{a} \sigma$, respectively. The robot continuously records measurements of the relative positions of the landmarks which are placed inside the trajectory circle, with standard deviation equal to $2 \%$ of the robot-to-landmark distance along each axis.

Fig. 1 shows the results for the robot orientation estimation errors in a typical realization. As evident, the errors of the standard EKF grow significantly faster than those of all other filters, which indicates that the standard EKF tends to diverge. Note also that although the orientation errors of the ideal EKF, FEJ-EKF, OC-EKF as well as the robocentric mapping filter remain well within their corresponding $3 \sigma$ bounds (computed from the square-root of the corresponding diagonal element 


\begin{tabular}{|c|c|c|c|c|}
\hline Ideal-EKF & Std-EKF & FEJ-EKF & OC-EKF & Robocentric \\
\hline \multicolumn{5}{|c|}{ Robot Position Err. RMS (m) } \\
\hline 0.6932 & 1.1406 & 0.7093 & 0.6977 & 0.8111 \\
\hline \multicolumn{5}{|c|}{ Robot Heading Err. RMS (rad) } \\
\hline 0.0634 & 0.0956 & 0.0671 & 0.0641 & 0.0716 \\
\hline \multicolumn{5}{|c|}{ Robot Pose NEES } \\
\hline 3.4643 & 18.5585 & 4.4979 & 3.8850 & 7.9436 \\
\hline \multicolumn{5}{|c|}{ Landmark Position Err. RMS (m) } \\
\hline 0.7377 & 1.2554 & 0.7558 & 0.7387 & 0.8726 \\
\hline \multicolumn{5}{|c|}{ Landmark Position NEES } \\
\hline 2.2647 & 18.4959 & 3.4480 & 2.9949 & 7.0308 \\
\hline
\end{tabular}

TABLE I

Robot Pose and Landmark Position Estimation Performance

of the estimated covariance matrix), those of the standard EKF exceed them. Most importantly, the $3 \sigma$ bounds of the standard EKF continuously decrease over time, as if the robot orientation was observable. However, the robot has no access to any new absolute orientation information (beyond what is available by re-observing the same two landmarks), and thus its orientation covariance should not continuously decrease at steady state. The results of Fig. 1 further strengthen our claim that in contrast to the ideal EKF, FEJ-EKF, OC-EKF, and robocentric mapping filter (cf. Sections IV-B1, V-A, V-B, and VI-C), the incorrect observability properties of the standard EKF cause an unjustified reduction in the orientation uncertainty.

\section{B. Second Simulation: Loop Closure}

To further test the performance of the five estimators, we conducted 50 Monte Carlo simulations in a SLAM scenario with loop closure. In this scenario, a robot executes 10 loops on a circular trajectory and observes 20 landmarks in total. For the results presented in the following, identical robot and sensor models to the preceding simulation (cf. Section VI-A) are used, while different sensor noise characteristics are employed. Specifically, the standard deviation for each wheel of the robot is equal to $\sigma=2 \% v$, while the standard deviation of the relative-position measurements is equal to $12 \%$ of the robotto-landmark distance along each axis. Moreover, the robot now only observes the landmarks that lie within its sensing range of $5 \mathrm{~m}$. It should be pointed out that the sensor-noise levels selected for this simulation are larger than what is typically encountered in practice. This was done purposefully, since higher noise levels lead to larger estimation errors, which make the effects of inconsistency more apparent.

The comparative results for all filters are presented in Fig. 2 and Table I. Specifically, Fig. 2(a) and Fig. 2(b) show the average NEES and RMS errors for the robot pose, respectively, versus time. On the other hand, Table I presents the average values of all relevant performance metrics for both the landmarks and the robot. As evident, the performance of the FEJ-EKF and the OC-EKF is very close to that of the ideal EKF, and substantially better than that of the standard EKF, both in terms of RMS errors and NEES. This occurs even though the Jacobians used in the FEJ-EKF and OCEKF are less accurate than those used in the standard EKF, as explained in the preceding section. This fact indicates that the errors introduced by the use of inaccurate Jacobians have a less detrimental effect on consistency and accuracy than the use of an error-state system model with incorrect observability properties. Moreover, it is important to note that the performance of the OC-EKF is superior to that of the FEJ-EKF by a small margin. This is attributed to the fact that the FEJ-EKF has larger linearization errors than the OCEKF, since the OC-EKF is optimal by construction, in terms of linearization errors, under the observability constraints.

\section{Comparison to Robocentric Mapping Filter}

From the plots of Fig. 2, we clearly see that both the FEJEKF and the OC-EKF also perform better than the robocentric mapping filter [Castellanos et al., 2004], [Castellanos et al., 2007], both in terms of accuracy and consistency. This result cannot be justified based on the observability properties of the filters: in [Castellanos et al., 2004], [Castellanos et al., 2007], the landmarks are represented in the robot frame, which can be shown to result in a system model with 3 unobservable degrees of freedom [Huang et al., 2008c]. However, in the robocentric mapping filter, during each propagation step all landmark position estimates need to be changed, since they are expressed with respect to the moving robot frame. As a result, during each propagation step (termed composition in [Castellanos et al., 2004], [Castellanos et al., 2007]), all landmark estimates and their covariance are affected by the linearization errors of the process model. This problem does not exist in the world-centric formulation of SLAM, and it could offer an explanation for the observed behavior.

To test this argument, we first examine the Kullback-Leibler divergence (KLD), between the pdf estimated by each filter, and the pdf estimated by its "ideal" counterpart. Specifically, we compute the KLD (i) between the pdf computed by the FEJ-EKF and that of the ideal EKF, (ii) between the pdf computed by the OC-EKF and that of the ideal EKF, and (iii) between the pdf computed by the robocentric mapping filter and that produced by an "ideal" robocentric mapping filter, which employs the true states in computing all the Jacobian matrices. The KLD is a standard measure for the difference between probability distributions. It is nonnegative, and equals zero only if the two distributions are identical [Cover and Thomas, 1991]. By computing the KLD between the estimated pdf and that of the "ideal" filter in each case, we can evaluate how close each filter is to its respective "golden standard". These results pertain to the same simulation setup presented in Section VI-B.

Since the five filters considered here (i.e., the OC-EKF, the FEJ-EKF, the ideal EKF, the robocentric mapping filter, and the ideal robocentric mapping filter) employ a Gaussian approximation of the pdf, we can compute the KLD in closed form. Specifically, the KLD from an approximation distribution, $p_{a}(\mathbf{x})=\mathcal{N}\left(\boldsymbol{\mu}_{a}, \mathbf{P}_{a}\right)$, to the ideal distribution, 

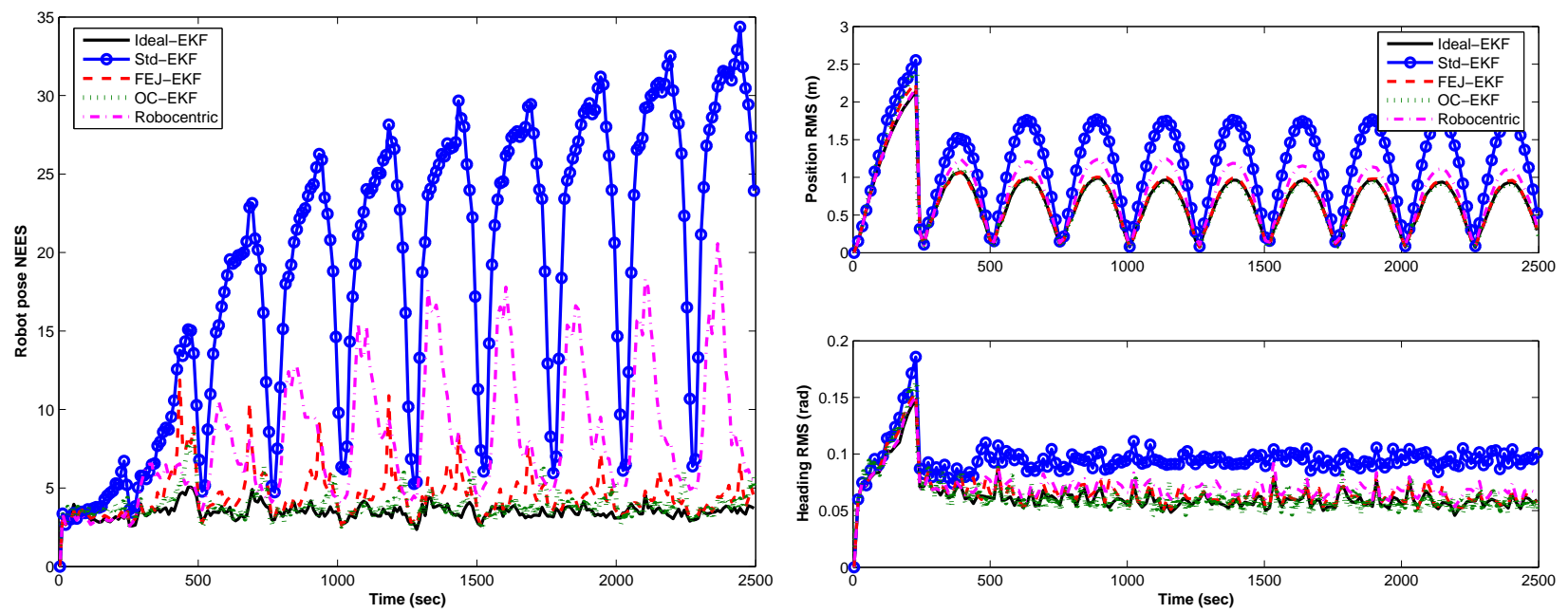

Fig. 2. Monte Carlo results for a SLAM scenario with multiple loop closures. (a) Average NEES of the robot pose errors (b) RMS errors for the robot pose (position and orientation). In these plots, the solid lines correspond to the ideal EKF, the dashed lines to the FEJ-EKF, the dotted lines to the OC-EKF, the solid lines with circles to the standard EKF, and the dash-dotted lines to the robocentric mapping filter of [Castellanos et al., 2004], [Castellanos et al., 2007]. Note that the RMS errors of the ideal EKF, FEJ-EKF, and OC-EKF are almost identical, which makes the corresponding lines difficult to distinguish.

$p_{o}(\mathbf{x})=\mathcal{N}\left(\boldsymbol{\mu}_{o}, \mathbf{P}_{o}\right)$, is given by:

$$
\begin{aligned}
d_{K L}= & \frac{1}{2}\left(\ln \left(\frac{\operatorname{det}\left(\mathbf{P}_{o}\right)}{\operatorname{det}\left(\mathbf{P}_{a}\right)}\right)+\operatorname{tr}\left(\mathbf{P}_{o}^{-1} \mathbf{P}_{a}\right)\right. \\
& \left.+\left(\boldsymbol{\mu}_{o}-\boldsymbol{\mu}_{a}\right)^{T} \mathbf{P}_{o}^{-1}\left(\boldsymbol{\mu}_{o}-\boldsymbol{\mu}_{a}\right)-\operatorname{dim}(\mathbf{x})\right)
\end{aligned}
$$

Fig. 3 presents the KLD over time, between the Gaussian distributions computed by the robocentric mapping filter, the FEJEKF and the OC-EKF, and those computed by their respective ideal filters (note that the $y$-axis scale is logarithmic). It is evident that the KLD in the case of the robocentric mapping filter is orders of magnitude larger than in the cases of the FEJEKF and the OC-EKF. This indicates that the linearization errors in the robocentric mapping filter result in a worse approximation of the ideal pdf.

We attribute this fact to the structure of the filter Jacobians. During the update step, the structure of the Jacobians in both the robocentric and the world-centric formulations is quite similar [Huang et al., 2008c]. In both cases, the terms appearing in the measurement Jacobians are either rotation matrices, or the robot-to-landmark position vector. However, the Jacobians employed during the composition step in the robocentric mapping filter are substantially more complex than those appearing in the world-centric EKF propagation (cf. (6)). Specifically, in the robocentric mapping filter, the state vector is given by (assuming a single landmark for simplicity):

$$
{ }^{R_{k}} \mathbf{x}_{k}=\left[\begin{array}{lll}
{ }^{R_{k}} \mathbf{p}_{G}^{T} & { }^{R_{k}} \phi_{G} & { }^{R_{k}} \mathbf{p}_{L}^{T}
\end{array}\right]^{T}
$$

The composition step is described by the following equations:

$$
\begin{aligned}
{ }^{R} \hat{\mathbf{p}}_{G} & =\mathbf{C}^{T}\left({ }^{R_{k-1}} \hat{\phi}_{R_{k}}\right)\left({ }^{R_{k-1}} \hat{\mathbf{p}}_{G}-{ }^{R_{k-1}} \hat{\mathbf{p}}_{R_{k}}\right) \\
{ }^{R_{k}} \hat{\phi}_{G} & ={ }^{R_{k-1}} \hat{\phi}_{G}-{ }^{R_{k-1}} \hat{\phi}_{R_{k}} \\
{ }^{{ }^{R}} \hat{\mathbf{p}}_{L} & =\mathbf{C}^{T}\left({ }^{R_{k-1}} \hat{\phi}_{R_{k}}\right)\left({ }^{R_{k-1}} \hat{\mathbf{p}}_{L}-{ }^{R_{k-1}} \hat{\mathbf{p}}_{R_{k}}\right)
\end{aligned}
$$

where ${ }^{R_{\ell}} \hat{\mathbf{p}}_{L}$ is the estimate of the landmark position with respect to the robot frame at time-step $\ell(\ell=k-1, k)$,

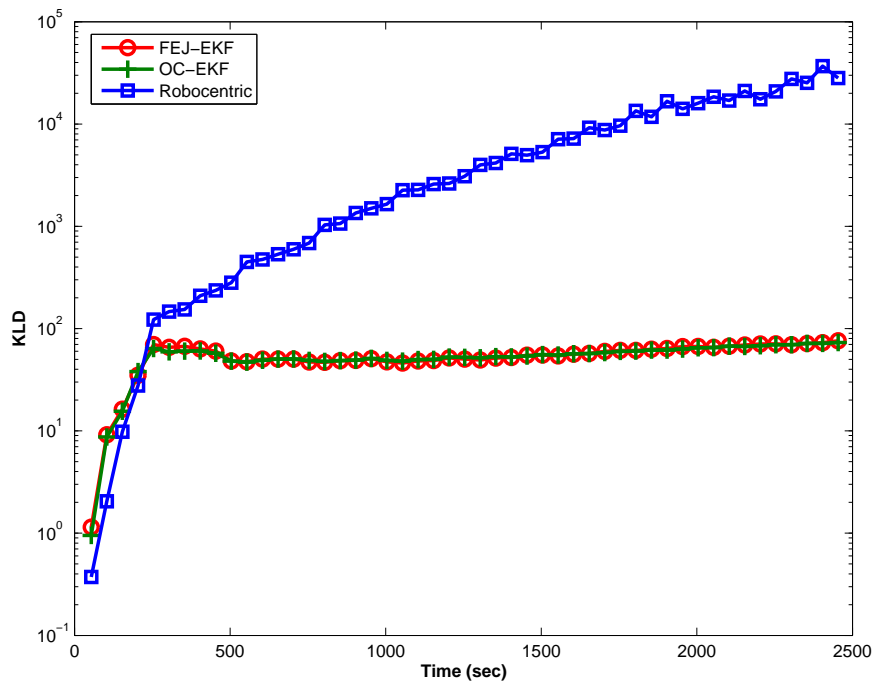

Fig. 3. Comparison results of the KLD in the SLAM scenario with multiple loop closures. In this plot, the solid line with circles corresponds to the FEJEKF, the solid line with crosses to the OC-EKF, and the solid line with squares to the robocentric mapping filter [Castellanos et al., 2004]. Note that the $y$ axis scale is logarithmic. Note that the KLD of the FEJ-EKF and OC-EKF are almost identical, which makes the corresponding lines difficult to distinguish.

$\left\{{ }^{R_{k-1}} \hat{\mathbf{p}}_{R_{k}},{ }^{R_{k-1}} \hat{\phi}_{R_{k}}\right\}$ is the estimate for the robot pose change between time-steps $k-1$ and $k$, expressed with respect to the robot frame at time-step $k-1$, and $\left\{{ }^{R_{\ell}} \mathbf{p}_{G},{ }^{R_{\ell}} \hat{\phi}_{G}\right\}$ is the estimate for the transformation between the robot frame and the global frame at time-step $\ell$. The linearized error propagation equation is given by:

$$
\left[\begin{array}{c}
R_{k} \widetilde{\mathbf{p}}_{G} \\
\widetilde{R}_{k}^{R_{k}} \\
{ }^{R_{k}} \widetilde{\mathbf{p}}_{L}
\end{array}\right]=\mathbf{J}_{L}^{R_{k-1}} \widetilde{\mathbf{p}}_{L}+\mathbf{J}_{G}\left[\begin{array}{c}
R_{k-1} \widetilde{\mathbf{p}}_{G} \\
R_{k-1} \widetilde{\phi}_{G}
\end{array}\right]+\mathbf{J}_{R}\left[\begin{array}{c}
R_{k-1} \widetilde{\mathbf{p}}_{R_{k}} \\
R_{k-1} \widetilde{\phi}_{R_{k}}
\end{array}\right]
$$



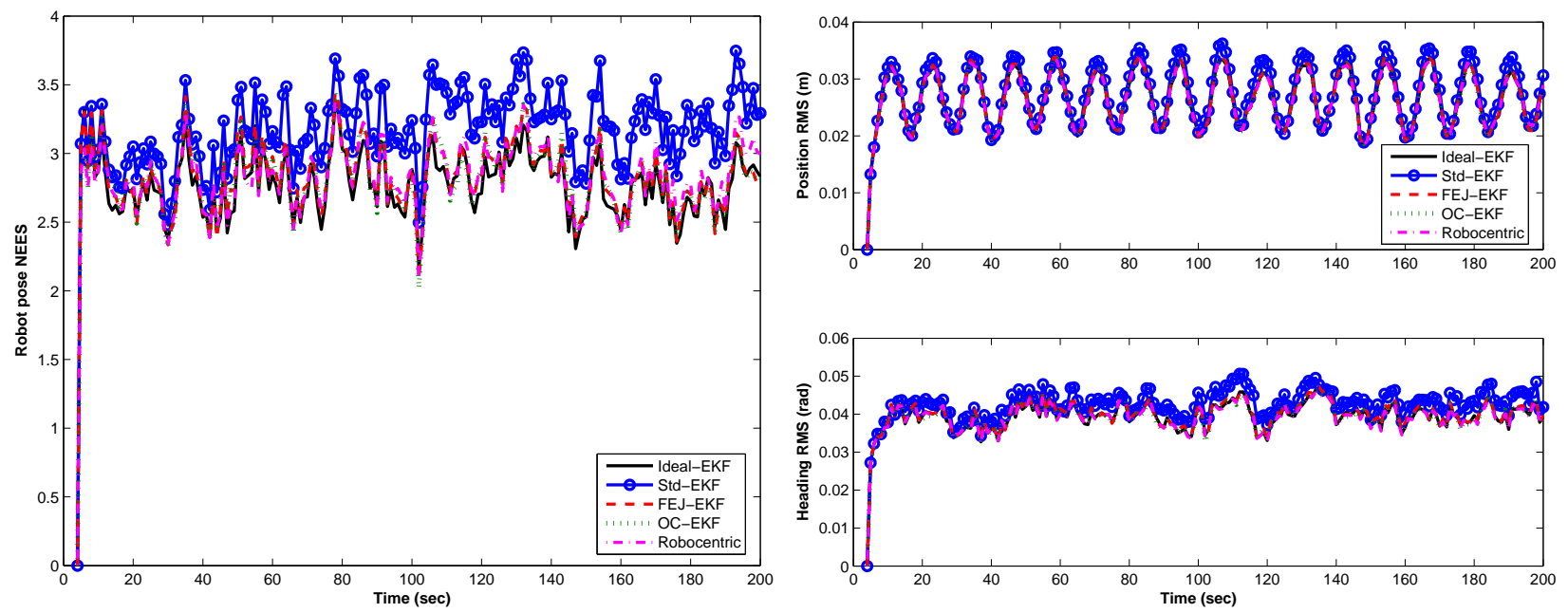

Fig. 4. Monte Carlo results for a "mini-SLAM" scenario with multiple loop closures where the robot trajectory and all landmarks are confined within a very small area of $1 \mathrm{~m} \times 1 \mathrm{~m}$. (a) Average NEES of the robot pose errors (b) RMS errors for the robot pose (position and orientation). In these plots, the solid lines correspond to the ideal EKF, the dashed lines to the FEJ-EKF, the dotted lines to the OC-EKF, the solid lines with circles to the standard EKF, and the dash-dotted lines to the robocentric mapping filter of [Castellanos et al., 2004]. Note that in this case both the NEES and the RMS errors of the ideal EKF, FEJ-EKF, OC-EKF, and the robocentric mapping filter are almost identical, which makes the corresponding lines difficult to distinguish.

where

$$
\begin{aligned}
& \mathbf{J}_{L}=\left[\begin{array}{c}
\mathbf{0}_{3 \times 2} \\
\mathbf{C}^{T}\left(R_{k-1} \hat{\phi}_{R_{k}}\right)
\end{array}\right], \mathbf{J}_{G}=\left[\begin{array}{cc}
\mathbf{C}^{T}\left(R_{k-1} \hat{\phi}_{R_{k}}\right) & \mathbf{0}_{2 \times 1} \\
\mathbf{0}_{1 \times 2} & 1 \\
\mathbf{0}_{2 \times 2} & \mathbf{0}_{2 \times 1}
\end{array}\right] \\
& \mathbf{J}_{R}=\left[\begin{array}{cc}
-\mathbf{C}^{T}\left(R_{k-1} \hat{\phi}_{R_{k}}\right) & -\mathbf{J}^{R_{k}} \hat{\mathbf{p}}_{G} \\
\mathbf{0}_{1 \times 2} & -1 \\
-\mathbf{C}^{T}\left({ }^{R_{k-1}} \hat{\phi}_{R_{k}}\right) & -\mathbf{J}^{R_{k}} \hat{\mathbf{p}}_{L}
\end{array}\right]
\end{aligned}
$$

We note that the state estimates appear in the Jacobian matrices $\mathbf{J}_{L}$ and $\mathbf{J}_{G}$ only through the rotation matrix $\mathbf{C}\left({ }^{R_{k-1}} \hat{\phi}_{R_{k}}\right)$. As a result, the difference between the ideal and actual Jacobians, $\mathbf{J}_{L}-\breve{\mathbf{J}}_{L}$ and $\mathbf{J}_{G}-\breve{\mathbf{J}}_{G}$ will only contain terms of the form $c\left({ }^{R_{k-1}} \hat{\phi}_{R_{k}}\right)-c\left({ }^{R_{k-1}} \phi_{R_{k}}\right)$, and $s\left({ }^{R_{k-1}} \hat{\phi}_{R_{k}}\right)-s\left({ }^{R_{k-1}} \phi_{R_{k}}\right)$. The magnitude of these terms is in the same order as ${ }^{R_{k-1}} \widetilde{\phi}_{R_{k}}$, which is typically a very small quantity. Thus, the discrepancy between the actual and ideal Jacobians is expected to be very small for $\mathbf{J}_{L}$ and $\mathbf{J}_{G}$.

On the other hand, in $\mathbf{J}_{R}$ the estimates for the landmark position and for the origin of the global frame with respect to the robot appear as well. As a result, the difference $\mathbf{J}_{R}-\breve{\mathbf{J}}_{R}$ will also contain the terms ${ }^{R_{k}} \widetilde{\mathbf{p}}_{G}$ and ${ }^{R_{k}} \widetilde{\mathbf{p}}_{L}$, whose magnitude can be significantly larger, e.g., in the order of meters (cf. Fig. 2). Thus, the Jacobian $\mathbf{J}_{R}$ can be very inaccurate. In contrast, the propagation Jacobians in the worldcentric formulation contain terms depending on (i) the robot's displacement between consecutive time steps, and (ii) the rotation matrix of the robot's orientation (cf. (8) and (9)). Since both of these quantities can be estimated with small errors, the world-centric EKF Jacobians are significantly more accurate than those of the robocentric formulation.

To further test this argument, we ran a simulation of a "miniSLAM" scenario, where both the robot trajectory and the landmarks are confined within a small area of $1 \mathrm{~m} \times 1 \mathrm{~m}$ (while all other settings are identical to the preceding simulation). In this setup, the estimation errors ${ }^{R_{k}} \widetilde{\mathbf{p}}_{G}$ and ${ }^{R_{k}} \widetilde{\mathbf{p}}_{L}$ remain small, and thus the Jacobians of the robocentric mapping filter become more accurate. The plots of Fig. 4 show the average NEES and RMS errors for the robot pose in this scenario. Interestingly, we observe that in this case the performance of the FEJ-EKF, the OC-EKF, and the robocentric mapping filter are almost identical. This validates the preceding discussion, and indicates that the representation used in the robocentric mapping filter results in performance loss in the case of large environments. This may justify the fact that the FEJ-EKF and OC-EKF outperform the algorithm of [Castellanos et al., 2004], even though all three filters employ a system model with three unobservable degrees of freedom.

As a final remark, we note that, in comparison to the FEJEKF and OC-EKF, the computational cost of the robocentric mapping filter is significantly higher. Specifically, both the FEJ-EKF and the OC-EKF have computational cost identical to the standard world-centric SLAM algorithm: linear in the number of landmarks during propagation, and quadratic during updates. On the other hand, both the update and the composition steps in the robocentric mapping filter have computational cost quadratic in the number of features, which results in approximately double overall computational burden.

\section{EXPERIMENTAL RESULTS}

Two sets of real-world experiments were performed to further test the proposed FEJ-EKF and OC-EKF algorithms. The results are presented next.

\section{A. First Experiment: Indoors}

The first experiment was conducted in an indoor office environment. The robot was commanded to perform 10 loops around a square with sides approximately equal to $20 \mathrm{~m}$ (cf. Fig. 5). This special trajectory was selected since repeated re-observation of the same landmarks tends to make the effects of inconsistency more apparent, and facilitates discerning the performance of the various filters. A Pioneer robot equipped 


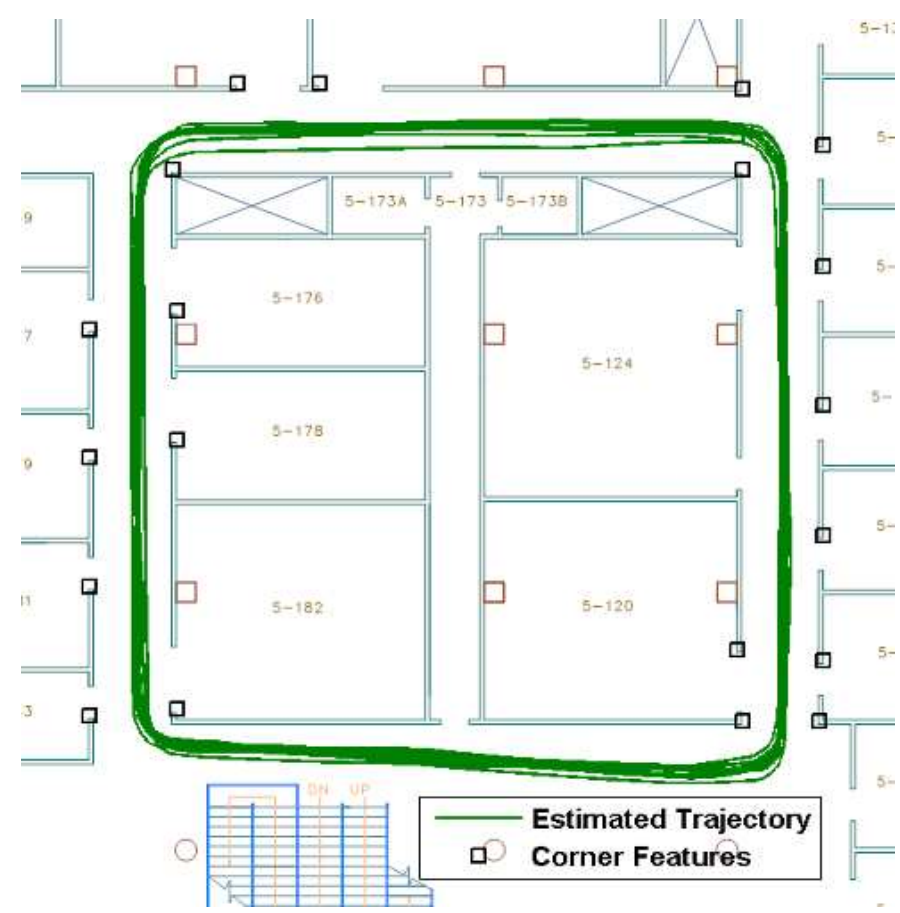

Fig. 5. The MAP estimate of the robot trajectory in the indoor experiment (solid line), overlaid on the blueprint of the building. The boxes ( $\square$ ) denote the corners whose exact location is known from the building's blueprint.
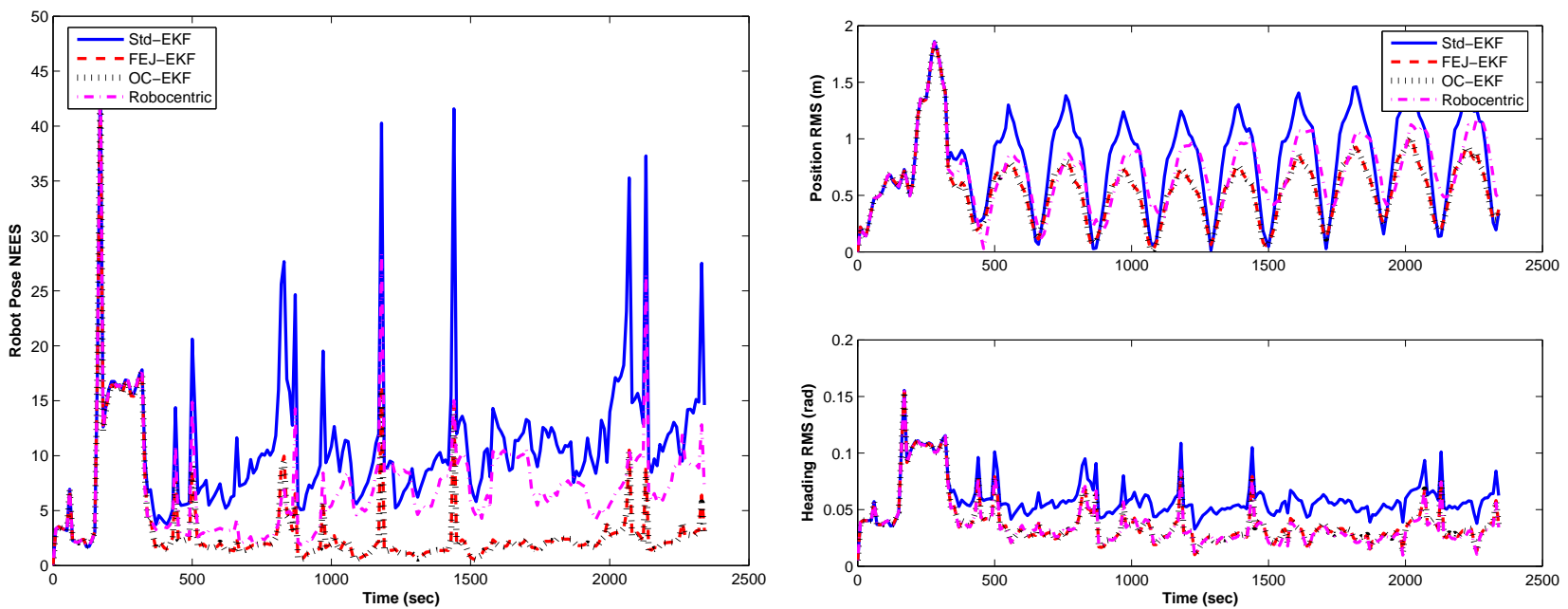

Fig. 6. (a) NEES of the robot pose errors (b) RMS errors for the robot pose (position and orientation). In these plots, the solid lines correspond to the standard EKF, the dashed lines to the FEJ-EKF, and the dotted lines to the OC-EKF, the dash-dotted lines to the robocentric mapping filter of [Castellanos et al., 2004]. Note that both the NEES and the RMS errors of the FEJ-EKF and OC-EKF are almost identical, which makes the corresponding lines difficult to distinguish.

with a SICK LMS200 laser range-finder and wheel encoders was used in this experiment. From the laser range data, corner features were extracted and used as landmarks, while the wheel encoders provided the linear and rotational velocity measurements. Propagation was carried out using the kinematic model described in Appendix A.

Because the ground truth of the robot pose could not be obtained using external sensors (e.g., overhead cameras), in this experiment, we obtained a reference trajectory by utilizing the known map of the area where the experiment took place. Specifically, the exact location of 20 corners was known from the blueprints of the building. Measurements to these corners, as well as all other measurements obtained by the robot (including to corners whose location was not known $a$ priori), were processed using a batch maximum a posteriori (MAP) estimator, to obtain an accurate estimate of the entire trajectory. This estimate, as well as the locations of the known corners, are shown in Fig. 5. This constitutes the ground truth against which the performance of the following filters was compared: (1) the standard EKF, (2) the FEJ-EKF, (3) the OC-EKF, and (4) the robocentric mapping filter. Clearly, due to the way the ground truth is computed, the filter errors are expected to have some correlation to the errors in the ground truth. However, since these correlations are the same for all 


\begin{tabular}{cccc}
\hline \hline Std-EKF & FEJ-EKF & OC-EKF & Robocentric \\
\hline \hline \multicolumn{3}{c}{ Robot Position Err. RMS (m) } \\
\hline 0.8209 & 0.5748 & 0.5754 & 0.7160 \\
\hline \hline \multicolumn{3}{c}{ Robot Heading Err. RMS (rad) } \\
\hline 0.0604 & 0.0397 & 0.0397 \\
\hline \hline \multicolumn{3}{c}{ Robot Pose NEES } \\
\hline 11.0706 & 3.5681 & 3.5282 \\
\hline \hline \multicolumn{3}{c}{ Landmark Position Err. RMS (m) } \\
\hline 1.1041 & 0.8675 & 0.8680 \\
\hline \hline \multicolumn{3}{c}{ Landmark Position NEES } \\
\hline 8.5033 & 5.9821 & 5.9836 \\
\hline \hline \multicolumn{3}{c}{ TABLE II } \\
RoBOT PoSE AND LANDMARK POSITION ESTIMATION PERFORMANCE
\end{tabular}

four filters, we can still have a fair comparison of their relative performance.

The results of NEES and RMS errors for all filters are presented in Figs. 6(a) and 6(b) and Table II. We point out that during the experiment the robot detected a number of features that were not included in the set of 20 known corners (e.g., movable objects such as furniture). Since no ground truth is available for the position of these objects, we only used the 20 known corners for computing the landmarks' error statistics. From the experimental results it becomes clear that in this particular experiment both the FEJ-EKF and OC-EKF outperform the standard EKF and the robocentric mapping filter, and perform almost identically to each other. This agrees with the simulation results presented in the preceding section.

\section{B. Second Experiment: Outdoors}

In the second experiment, the performance of the FEJ-EKF and OC-EKF was tested on the Sydney Car Park data set collected by Guivant and $\mathrm{Nebot}^{4}$. The experimental platform is a 4-wheeled vehicle equipped with a GPS receiver, a laser sensor, and wheel encoders. The kinematic GPS system was used to provide ground truth for the robot position with $5 \mathrm{~cm}$ accuracy. Since the GPS has different frequency (up to $2 \mathrm{~Hz}$ ) from the other sensors, we interpolated the GPS data to obtain the ground truth at each time step. Wheel encoders were used to provide odometric measurements, and propagation was carried out using the Ackerman model. In this particular application, $60 \mathrm{~mm}$ steel poles covered with reflective tape were used artificial landmarks. With this approach, it is easy to extract the features and the measurement model becomes very accurate. Since the true position of the landmarks was also obtained with GPS, a true map was available for comparison purposes.

In this test, because the ground truth for the robot orientation was still unavailable, the ideal EKF could not be tested, and therefore the same filters as in the first experiment were compared: (1) the standard EKF, (2) the FEJ-EKF, (3) the OC-EKF, and (4) the robocentric mapping filter. The comparison results are shown in Table III, and Figs. 7 and 8. Specifically, Table III

\footnotetext{
${ }^{4}$ The data set is available at: www-personal.acfr.usyd.edu.au/nebot/dataset
}

\begin{tabular}{cccc}
\hline \hline Std-EKF & FEJ-EKF & OC-EKF & Robocentric \\
\hline \hline \multicolumn{4}{c}{ Robot Position Err. RMS (m) } \\
\hline 0.1002 & 0.0523 & 0.0522 & 0.0838 \\
\hline \multicolumn{4}{c}{ Robot Position NEES } \\
\hline 2.8900 & 2.5197 & 2.4705 \\
\hline \multicolumn{4}{c}{ Landmark Position Err. RMS (m) } \\
\hline 0.3812 & 0.1858 & 0.1860 & 0.2755 \\
\hline \hline \multicolumn{4}{c}{ Landmark Position NEES } \\
\hline 2.5196 & 2.0197 & 1.9818 \\
\hline \hline
\end{tabular}

TABLE III

Robot and Landmark Position Estimation Performance

presents the average values of all relevant performance metrics for the robot and the landmarks. On the other hand, Fig. 7 shows the trajectory and landmark estimates produced by the four filters, while Fig. 8 shows the NEES and RMS errors of the robot position over time. We point out that the NEES in this case pertains only to the robot position, and therefore the "optimal" value for it is 2 .

Similarly to the results presented in the first experiment, this test also demonstrates that both the FEJ-EKF and OC-EKF outperform the standard EKF and the robocentric mapping filter, and perform very close to each other. In particular, the average RMS errors and the average NEES for the FEJEKF and OC-EKF are smaller than the corresponding ones for the two competing filters. These results, along with those of the simulations presented in the previous section, support our conjecture, which states that the mismatch in the dimension of the unobservable subspace between the linearized SLAM system and the underlying nonlinear system is a fundamental cause of filter inconsistency.

\section{SUMMARY}

In this paper, we have presented an observability-based study of the inconsistency problem in EKF-based SLAM. By comparing the observability properties of the nonlinear SLAM system model with those of the linearized error-state model employed in the EKF, we proved that the observable subspace of the standard EKF is always of higher dimension than the observable subspace of the underlying nonlinear system. As a result, the covariance estimates of the EKF undergo reduction in directions of the state space where no information is available, which is a primary cause of inconsistency. Based on the above analysis, we have proposed a new methodology for the design of EKF-based estimators for SLAM. Our approach dictates selecting the linearization points of the EKF so as to ensure that the resulting linearized system model has three unobservable directions.

We propose two filters, the First Estimates Jacobian (FEJ)EKF and the Observability Constrained (OC)-EKF, which adhere to the above design methodology. Specifically, in the FEJ-EKF all Jacobians are calculated using the first available estimate for each state variable, while in the OC-EKF the linearization points are obtained in closed form by solving 


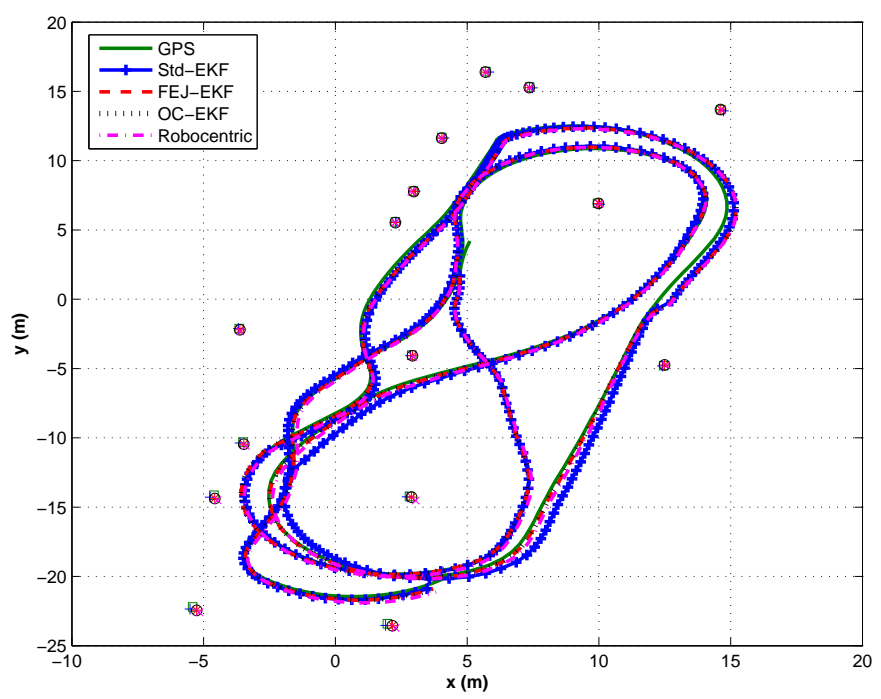

Fig. 7. The robot trajectory and landmark estimates. In this plot, the solid line depicts the ground truth obtained from GPS, while the boxes ( $\square$ ) are the known beacon positions. The dashed line with crosses and the crosses $(+)$ denote the estimated trajectory and landmarks, respectively, corresponding to the standard EKF, the dashed line and stars (*) correspond to the FEJ-EKF, the dotted line and circles (o) to the OC-EKF, and the dash-dotted line and x-crosses $(\times)$ to the robocentric mapping filter of [Castellanos et al., 2004].
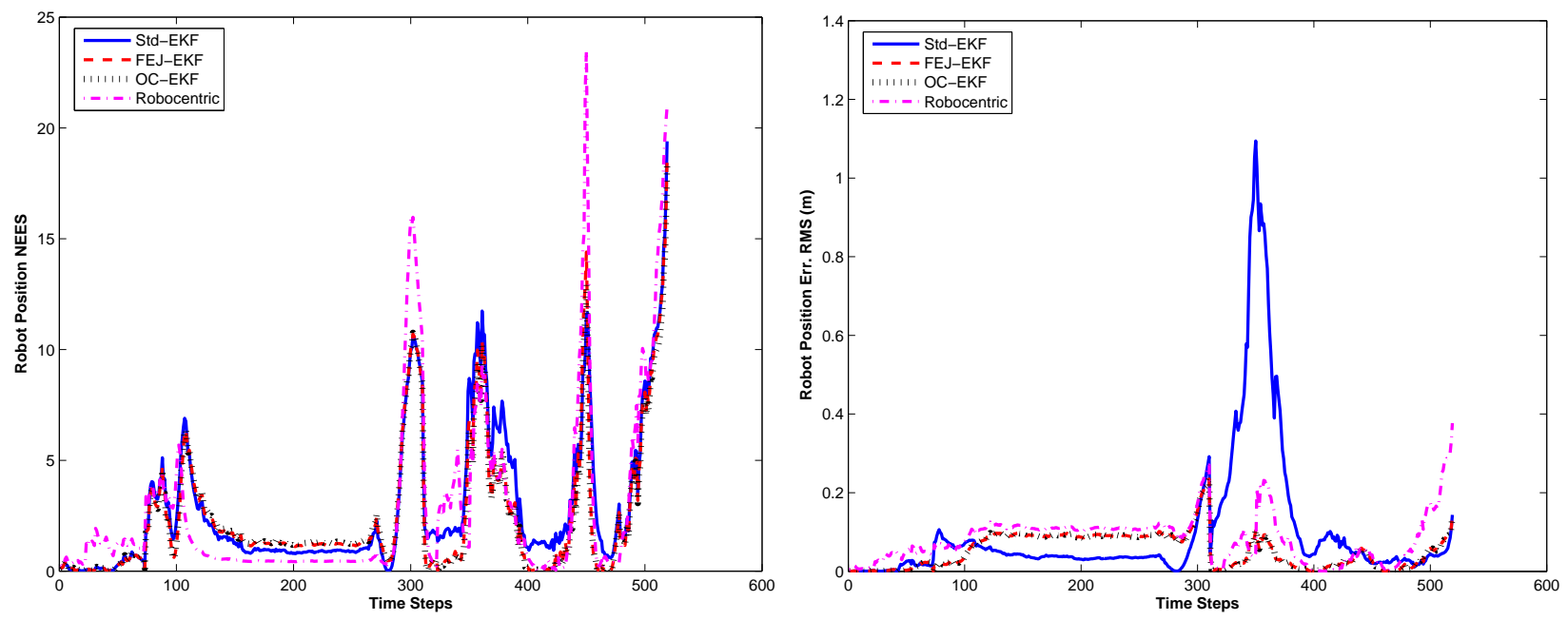

Fig. 8. (a) NEES of the robot position errors (b) RMS errors for the robot position. In these plots, the solid lines correspond to the standard EKF, the dashed lines to the FEJ-EKF, the dotted lines to the OC-EKF, and the dash-dotted lines to the robocentric mapping filter of [Castellanos et al., 2004]. Note that both the NEES and the RMS errors of the FEJ-EKF and OC-EKF are almost identical, which makes the corresponding lines difficult to distinguish.

an observability-constrained minimization problem (i.e., minimizing the expected linearization errors subject to the observability constraints). As a result, the linearized system models employed in these two filters have the desirable observability properties. Extensive simulation and experimental tests verify that the FEJ-EKF and the OC-EKF perform significantly better, in terms of both accuracy and consistency, than the standard EKF and the robocentric mapping filter. This occurs despite the fact that the Jacobians used in the FEJ-EKF and OC-EKF are evaluated using less accurate linearization points. These results indicate that ensuring the correct observability properties of the linearized system model is a crucial requirement.

\section{APPENDIX A UNICYCLE MODEL}

If the unicycle model is used, and we employ the approximation that the velocity and heading are constant during each propagation interval, we obtain ${ }^{R_{k}} \hat{\mathbf{x}}_{R_{k+1}}=$ $\left[\begin{array}{lll}v_{m_{k}} \delta t & 0 & \omega_{m_{k}} \delta t\end{array}\right]^{T}$, where $\mathbf{u}_{m_{k}}=\left[\begin{array}{ll}v_{m_{k}} & \omega_{m_{k}}\end{array}\right]^{T}$ are the linear and rotational velocity measurements, respectively, and $\delta t$ is the sampling period. Substitution in (2)-(3) yields the familiar robot pose propagation equations:

$$
\begin{aligned}
\hat{\mathbf{p}}_{R_{k+1 \mid k}} & =\hat{\mathbf{p}}_{R_{k \mid k}}+\left[\begin{array}{l}
v_{m_{k}} \delta t c\left(\hat{\phi}_{R_{k \mid k}}\right) \\
v_{m_{k}} \delta t s\left(\hat{\phi}_{R_{k \mid k}}\right)
\end{array}\right] \\
\hat{\phi}_{R_{k+1 \mid k}} & =\hat{\phi}_{R_{k \mid k}}+\omega_{m_{k}} \delta t
\end{aligned}
$$

Similarly, the commonly used expressions for the Jacobian matrices $\boldsymbol{\Phi}_{R_{k}}$ and $\mathbf{G}_{R_{k}}$ can be derived from (6), (7) 
and (9). Specifically, by substituting the robot displacement ${ }^{R_{k}} \hat{\mathbf{p}}_{R_{k+1}}=\left[\begin{array}{ll}v_{m_{k}} \delta t & 0\end{array}\right]^{T}$ into (7), we have:

$$
\boldsymbol{\Phi}_{R_{k}}=\left[\begin{array}{ccc}
1 & 0 & -v_{m_{k}} \delta t s\left(\hat{\phi}_{R_{k \mid k}}\right) \\
0 & 1 & v_{m_{k}} \delta t c\left(\hat{\phi}_{R_{k \mid k}}\right) \\
0 & 0 & 1
\end{array}\right]
$$

To derive the Jacobian matrix $\mathbf{G}_{R_{k}}^{o}$ with respect to the odometry vector $\mathbf{u}_{k}$, instead of ${ }^{R_{k}} \hat{\mathbf{x}}_{R_{k+1}}$, we apply the chain rule of differentiation as follows:

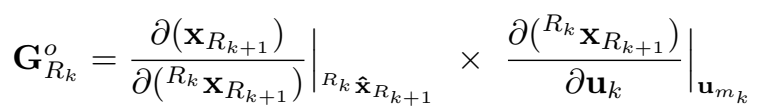

The first term is the Jacobian with respect to the robot pose change (displacement and orientation change), evaluated at the estimate ${ }^{R_{k}} \hat{\mathbf{x}}_{R_{k+1}}$, and is given in (9). The second term is the Jacobian of the robot pose change with respect to $\mathbf{u}_{k}$. Since ${ }^{R_{k}} \mathbf{x}_{R_{k+1}}=\left[\begin{array}{lll}v_{k} \delta t & 0 & \omega_{k} \delta t\end{array}\right]^{T}$, this Jacobian is simply given by:

$$
\left.\frac{\partial\left({ }^{R_{k}} \mathbf{x}_{R_{k+1}}\right)}{\partial \mathbf{u}_{k}}\right|_{\mathbf{u}_{m_{k}}}=\left[\begin{array}{cc}
\delta t & 0 \\
0 & 0 \\
0 & \delta t
\end{array}\right]
$$

Therefore, substitution of (68) and (9) into (67) yields:

$$
\mathbf{G}_{R_{k}}^{o}=\left[\begin{array}{cc}
\delta t c\left(\hat{\phi}_{R_{k \mid k}}\right) & 0 \\
\delta t s\left(\hat{\phi}_{R_{k \mid k}}\right) & 0 \\
0 & \delta t
\end{array}\right]
$$

We thus showed how the commonly used expressions for (2)(4), as well as the state and noise Jacobians can be derived.

\section{APPENDIX B}

\section{PROOF OF LEMMA 4.1}

The proof is based on mathematical induction, by verifying the structure of the $k$ th order Lie derivatives. We define the Lie derivative of a $C^{\infty}$ function $h$ on an open subset $\mathcal{S} \subset \mathbb{R}^{\operatorname{dim}(\mathbf{x})}$ along an analytic vector field $\mathbf{f}$ on $\mathcal{S}$, as:

$$
L_{\mathbf{f}} h=(d h) \mathbf{f}
$$

where $d h$ is the gradient of $h$ with respect to the state vector $\mathbf{x}$. We start by noting the following identities, which will be useful in the ensuing derivations.

$$
\begin{aligned}
\frac{d \rho}{d \mathbf{x}} & =\left[\begin{array}{lllll}
-\frac{\delta x}{\rho} & -\frac{\delta y}{\rho} & 0 & \frac{\delta x}{\rho} & \frac{\delta y}{\rho}
\end{array}\right] \\
& =\left[\begin{array}{lllll}
-c \theta & -s \theta & 0 & c \theta & s \theta
\end{array}\right] \\
\frac{d \psi}{d \mathbf{x}} & =\frac{1}{\rho}\left[\begin{array}{lllll}
\frac{\delta y}{\rho} & -\frac{\delta x}{\rho} & -\rho & -\frac{\delta y}{\rho} & \frac{\delta x}{\rho}
\end{array}\right] \\
& =\frac{1}{\rho}\left[\begin{array}{lllll}
s \theta & -c \theta & -\rho & -s \theta & c \theta
\end{array}\right]
\end{aligned}
$$

where $\delta x \triangleq x_{L}-x_{R}, \delta y \triangleq y_{L}-y_{R}$, and $\theta \triangleq \psi+\phi_{R}$.

We first prove that if $h$ has the special structure shown in (15), then the zeroth- and first-order Lie derivatives are functions of $\rho$ and $\psi$ only.

By applying the chain rule of differentiation, the zerothorder (i.e., $k=0$ ) Lie derivative is computed as follows:

$$
L^{0} h \triangleq \frac{d h}{d \mathbf{x}}=\underbrace{\left[\begin{array}{ll}
\frac{\partial h}{\partial \rho} & \frac{\partial h}{\partial \psi}
\end{array}\right]}_{\mathbf{A}_{0}}\left[\begin{array}{l}
\frac{d \rho}{d \mathbf{x}} \\
\frac{d \psi}{d \mathbf{x}}
\end{array}\right]
$$

It is important to note that since $h$ is a function of $\rho$ and $\psi$ only, the terms $\frac{\partial h}{\partial \rho}$ and $\frac{\partial h}{\partial \psi}$ are also functions of $\rho$ and $\psi$ only. As a result, the matrix $\mathbf{A}_{0}$ is a function of $\rho$ and $\psi$, whose exact structure depends on the particular measurement function $h$.

The first-order (i.e., $k=1$ ) Lie derivatives are calculated according to the definition (70), and employing the results of (71) and (72), as:

$$
\begin{aligned}
L_{\mathbf{f}_{1}}^{1} h & =\left[\begin{array}{ll}
\frac{\partial h}{\partial \rho} & \frac{\partial h}{\partial \psi}
\end{array}\right]\left[\begin{array}{l}
\frac{d \rho}{d \mathbf{x}} \\
\frac{d \psi}{d \mathbf{x}}
\end{array}\right] \mathbf{f}_{1} \\
& =\mathbf{A}_{0}\left[\begin{array}{c}
-c \theta c \phi_{R}-s \theta s \phi_{R} \\
\frac{1}{\rho}\left(s \theta c \phi_{R}-c \theta s \phi_{R}\right)
\end{array}\right]=\mathbf{A}_{0}\left[\begin{array}{c}
-c \psi \\
\frac{s \psi}{\rho}
\end{array}\right] \\
L_{\mathbf{f}_{2}}^{1} h & =\left[\begin{array}{ll}
\frac{\partial h}{\partial \rho} & \frac{\partial h}{\partial \psi}
\end{array}\right]\left[\begin{array}{l}
\frac{d \rho}{d \mathbf{x}} \\
\frac{d \psi}{d \mathbf{x}}
\end{array}\right] \mathbf{f}_{2}=\mathbf{A}_{0}\left[\begin{array}{c}
0 \\
-1
\end{array}\right]
\end{aligned}
$$

We thus see that both the zeroth- and the first-order Lie derivatives are functions of $\rho$ and $\psi$ only. This is the base case for the proof by induction.

Now assume the $k$-th order Lie derivatives $L_{\mathbf{f}_{i}}^{k} h, i=1,2$, are functions of $\rho$ and $\psi$ only. ${ }^{5}$ Then their gradients can be computed by:

$$
\frac{d\left(L_{\mathbf{f}_{i}}^{k} h\right)}{d \mathbf{x}}=\underbrace{\left[\begin{array}{ll}
\frac{\partial}{\partial \rho}\left(L_{\mathbf{f}_{i}}^{k} h\right) & \frac{\partial}{\partial \psi}\left(L_{\mathbf{f}_{i}}^{k} h\right)
\end{array}\right]}_{\mathbf{A}_{k_{i}}}\left[\begin{array}{l}
\frac{d \rho}{d \mathbf{x}} \\
\frac{d \psi}{d \mathbf{x}}
\end{array}\right]
$$

where $\mathbf{A}_{k_{i}}$ is a function of $\rho$ and $\psi$ only. Thus, the $(k+1)$-th order Lie derivatives are computed as follows:

$$
\begin{aligned}
L_{\mathbf{f}_{1}}^{k+1} h & =\left[\begin{array}{ll}
\frac{\partial}{\partial \rho}\left(L_{\mathbf{f}_{1}}^{k} h\right) & \frac{\partial}{\partial \psi}\left(L_{\mathbf{f}_{1}}^{k} h\right)
\end{array}\right]\left[\begin{array}{l}
\frac{d \rho}{d \mathbf{x}} \\
\frac{d \psi}{d \mathbf{x}}
\end{array}\right] \mathbf{f}_{1} \\
& =\mathbf{A}_{k_{1}}\left[\begin{array}{c}
-c \theta c \phi_{R}-s \theta s \phi_{R} \\
\frac{1}{\rho}\left(s \theta c \phi_{R}-c \theta s \phi_{R}\right)
\end{array}\right]=\mathbf{A}_{k_{1}}\left[\begin{array}{c}
-c \psi \\
\frac{s \psi}{\rho}
\end{array}\right] \\
L_{\mathbf{f}_{2}}^{k+1} h & =\left[\begin{array}{ll}
\frac{\partial}{\partial \rho}\left(L_{\mathbf{f}_{2}}^{k} h\right) & \frac{\partial}{\partial \psi}\left(L_{\mathbf{f}_{2}}^{k} h\right)
\end{array}\right]\left[\begin{array}{l}
\frac{d \rho}{d \mathbf{x}} \\
\frac{d \psi}{d \mathbf{x}}
\end{array}\right] \mathbf{f}_{2}=\mathbf{A}_{k_{2}}\left[\begin{array}{c}
0 \\
-1
\end{array}\right]
\end{aligned}
$$

Clearly, the $(k+1)$-th order Lie derivatives are also functions of $\rho$ and $\psi$ only, and the proof by induction is complete.

\footnotetext{
${ }^{5}$ Extension of this analysis to the case of mixed $k$-th order Lie derivatives is straightforward, though more involved in terms of notation; thus, it is omitted to preserve presentation clarity.
} 


\section{APPENDIX C}

PROOF OF LEMMA 4.2

Employing the expressions for the Lie derivatives derived in Appendix B, we have:

$$
\begin{aligned}
& d \mathcal{G}=\operatorname{span}\left\{\begin{array}{ccc}
\frac{d\left(L^{0} h_{1}\right)}{d \mathbf{x}}, & \ldots, & \frac{d\left(L^{0} h_{n}\right)}{d \mathbf{x}}, \\
\frac{d\left(L_{\mathbf{f}_{j}} h_{1}\right)}{d \mathbf{x}}, & \ldots, & \frac{d\left(L_{\mathbf{f}_{j}} h_{n}\right)}{d \mathbf{x}}, \\
\ldots & \ldots & \ldots \\
\frac{d\left(L_{\mathbf{f}_{j}}^{k} h_{1}\right)}{d \mathbf{x}}, & \ldots, & \frac{d\left(L_{\mathbf{f}_{j}}^{k} h_{n}\right)}{d \mathbf{x}}
\end{array}\right\} \\
& =\operatorname{span}\left\{\begin{array}{ccc}
\mathbf{A}_{0}^{1}\left[\begin{array}{l}
\frac{d \rho}{d \mathbf{x}} \\
\frac{d \psi}{d \mathbf{x}}
\end{array}\right], & \ldots, & \mathbf{A}_{0}^{n}\left[\begin{array}{c}
\frac{d \rho}{d \mathbf{x}} \\
\frac{d \psi}{d \mathbf{x}}
\end{array}\right], \\
\mathbf{A}_{1_{j}}^{1}\left[\begin{array}{l}
\frac{d \rho}{d \mathbf{x}} \\
\frac{d \psi}{d \mathbf{x}}
\end{array}\right], & \ldots, & \mathbf{A}_{1_{j}}^{n}\left[\frac{\frac{d \rho}{d \mathbf{x}}}{\frac{d \psi}{d \mathbf{x}}}\right], \\
\ldots & \ldots & \ldots \\
\mathbf{A}_{k_{j}}^{1}\left[\frac{\frac{d \rho}{d \mathbf{x}}}{\frac{d \psi}{d \mathbf{x}}}\right], & \ldots, & \mathbf{A}_{k_{j}}^{n}\left[\frac{\frac{d \rho}{d \mathbf{x}}}{\frac{d \psi}{d \mathbf{x}}}\right]
\end{array}\right\}
\end{aligned}
$$

where the index $j=1,2$ corresponds to the vectors $\mathbf{f}_{1}$ and $\mathbf{f}_{2}$, and the superscript $i$ in $\mathbf{A}^{i}$ to the measurement function $h_{i}$ (i.e., $i=1,2, \ldots, n$ ). Clearly, the row-span of all the above vectors is identical to the row-span of $\frac{d \rho}{d \mathbf{x}}$ and $\frac{d \psi}{d \mathbf{x}}$, i.e.,

$$
\begin{aligned}
d \mathcal{G} & =\underset{\text { row }}{\operatorname{span}}\left[\begin{array}{ccccc}
-c \theta & -s \theta & 0 & c \theta & s \theta \\
\frac{s \theta}{\rho} & -\frac{c \theta}{\rho} & -1 & -\frac{s \theta}{\rho} & \frac{c \theta}{\rho}
\end{array}\right] \\
= & \operatorname{span}_{\text {row }}\left\{\mathbf{J} \operatorname{Diag}\left(\frac{1}{\rho}, 1\right) \mathbf{C}^{T}(\psi)\right. \\
& \left.\times\left[\begin{array}{lllll}
s \phi_{R} & -c \phi_{R} & -c \phi_{R} \delta x-s \phi_{R} \delta y & -s \phi_{R} & c \phi_{R} \\
c \phi_{R} & s \phi_{R} & s \phi_{R} \delta x-c \phi_{R} \delta y & -c \phi_{R} & -s \phi_{R}
\end{array}\right]\right\} \\
= & \underset{\operatorname{row}}{\operatorname{span}}\left[\begin{array}{ccccc}
s \phi_{R} & -c \phi_{R} & -c \phi_{R} \delta x-s \phi_{R} \delta y & -s \phi_{R} & c \phi_{R} \\
c \phi_{R} & s \phi_{R} & s \phi_{R} \delta x-c \phi_{R} \delta y & -c \phi_{R} & -s \phi_{R}
\end{array}\right]
\end{aligned}
$$

\section{APPENDIX D}

\section{PROOF OF LEMMA 5.2}

Under the Gaussianity assumption, it is $p\left(\mathbf{x}_{R_{k}} \mid \mathbf{z}_{0: k}\right)=$ $\mathcal{N}\left(\hat{\mathbf{x}}_{R_{k \mid k}}, \mathbf{P}_{R R_{k \mid k}}\right)$, where $\mathbf{P}_{R R_{k \mid k}}$ is the covariance matrix corresponding to the robot pose, obtained by partitioning the state covariance matrix as $\mathbf{P}_{k \mid k}=\left[\begin{array}{ll}\mathbf{P}_{R R_{k \mid k}} & \mathbf{P}_{R L_{k \mid k}} \\ \mathbf{P}_{R L_{k \mid k}^{T}}^{T} & \mathbf{P}_{L L_{k \mid k}}\end{array}\right]$, and $p\left(\mathbf{x}_{k+1} \mid \mathbf{z}_{0: k}\right)=\mathcal{N}\left(\hat{\mathbf{x}}_{k+1 \mid k}, \mathbf{P}_{k+1 \mid k}\right)$.

The first term of the cost function (46) is computed as:

$$
\begin{aligned}
& \int\left\|\mathbf{x}_{R_{k}}-\mathbf{x}_{R_{k \mid k}}^{\star}\right\|^{2} p\left(\mathbf{x}_{R_{k}} \mid \mathbf{z}_{0: k}\right) d \mathbf{x}_{R_{k}} \\
= & \int\left(\mathbf{x}_{R_{k}}^{T} \mathbf{x}_{R_{k}}-2 \mathbf{x}_{R_{k}}^{T} \mathbf{x}_{R_{k \mid k}}^{\star}+\mathbf{x}_{R_{k \mid k}}^{\star T} \mathbf{x}_{R_{k \mid k}}^{\star}\right) p\left(\mathbf{x}_{R_{k}} \mid \mathbf{z}_{0: k}\right) d \mathbf{x}_{R_{k}} \\
= & \mathbb{E}\left(\mathbf{x}_{R_{k}}^{T} \mathbf{x}_{R_{k}}\right)-2 \mathbb{E}\left(\mathbf{x}_{R_{k}}^{T}\right) \mathbf{x}_{R_{k \mid k}}^{\star}+\mathbf{x}_{R_{k \mid k}}^{\star T} \mathbf{x}_{R_{k \mid k}}^{\star} \\
= & \operatorname{tr}\left(\mathbf{P}_{R R_{k \mid k}}+\hat{\mathbf{x}}_{R_{k \mid k}} \hat{\mathbf{x}}_{R_{k \mid k}}^{T}\right)-2 \hat{\mathbf{x}}_{R_{k \mid k}}^{T} \mathbf{x}_{R_{k \mid k}}^{\star}+\mathbf{x}_{R_{k \mid k}}^{\star T} \mathbf{x}_{R_{k \mid k}}^{\star} \\
= & \operatorname{tr}\left(\mathbf{P}_{R R_{k \mid k}}\right)+\hat{\mathbf{x}}_{R_{k \mid k}}^{T} \hat{\mathbf{x}}_{R_{k \mid k}}-2 \hat{\mathbf{x}}_{R_{k \mid k}}^{T} \mathbf{x}_{R_{k \mid k}}^{\star}+\mathbf{x}_{R_{k \mid k}}^{\star T} \mathbf{x}_{R_{k \mid k}} \\
= & \operatorname{tr}\left(\mathbf{P}_{R R_{k \mid k}}\right)+\left\|\hat{\mathbf{x}}_{R_{k \mid k}}-\mathbf{x}_{R_{k \mid k}}^{\star}\right\|^{2}
\end{aligned}
$$

where $\mathbb{E}(\cdot)$ denotes expectation and $\operatorname{tr}(\cdot)$ the matrix trace. Proceeding similarly, the second term of the cost function (46) can be derived as:

$$
\begin{aligned}
& \int\left\|\mathbf{x}_{k+1}-\mathbf{x}_{k+1 \mid k}^{\star}\right\|^{2} p\left(\mathbf{x}_{k+1} \mid \mathbf{z}_{0: k}\right) d \mathbf{x}_{k+1} \\
= & \operatorname{tr}\left(\mathbf{P}_{k+1 \mid k}\right)+\left\|\hat{\mathbf{x}}_{k+1 \mid k}-\mathbf{x}_{k+1 \mid k}^{\star}\right\|^{2}
\end{aligned}
$$

Using (79) and (80), as well as the fact that the true $\mathbf{P}_{R R_{k \mid k}}$ and $\mathbf{P}_{k+1 \mid k}$ are independent of the linearization points, the following equivalence is immediate:

$$
\begin{aligned}
\min _{\mathbf{x}_{R_{k \mid k}}^{\star}, \mathbf{x}_{k+1 \mid k}^{\star}}\left\{\begin{array}{l}
\operatorname{tr}\left(\mathbf{P}_{R R_{k \mid k}}\right)+\operatorname{tr}\left(\mathbf{P}_{k+1 \mid k}\right)+ \\
\left\|\hat{\mathbf{x}}_{R_{k \mid k}}-\mathbf{x}_{R_{k \mid k}}^{\star}\right\|^{2}+\left\|\hat{\mathbf{x}}_{k+1 \mid k}-\mathbf{x}_{k+1 \mid k}^{\star}\right\|^{2}
\end{array}\right\} \\
\Leftrightarrow \min _{\mathbf{x}_{R_{k \mid k}}^{\star}, \mathbf{x}_{k+1 \mid k}^{\star}}\left\|\hat{\mathbf{x}}_{R_{k \mid k}}-\mathbf{x}_{R_{k \mid k}}^{\star}\right\|^{2}+\left\|\hat{\mathbf{x}}_{k+1 \mid k}-\mathbf{x}_{k+1 \mid k}^{\star}\right\|^{2}
\end{aligned}
$$

We now derive the following identities for the observability constraint (47) (cf. (36) and (42)):

$$
\begin{aligned}
& \mathbf{H}_{k+1} \boldsymbol{\Phi}_{k} \cdots \boldsymbol{\Phi}_{k_{o}} \mathbf{U}=\mathbf{0} \\
\Leftrightarrow & \mathbf{H}_{L_{k+1}}\left[-\mathbf{I}_{2}-\mathbf{J}\left(\mathbf{p}_{L_{k+1 \mid k}}^{\star}-\mathbf{p}_{R_{k_{o} \mid k_{o}}}^{\star}-\sum_{j=k_{o}+1}^{k} \Delta \mathbf{p}_{R_{j}}^{\star}\right) \mathbf{I}_{2}\right] \mathbf{U}=\mathbf{0} \\
\Leftrightarrow & \mathbf{p}_{L_{k+1 \mid k}}^{\star}-\mathbf{p}_{R_{k \mid k}}^{\star}=\hat{\mathbf{p}}_{L_{k_{o} \mid k_{o}}}-\mathbf{p}_{R_{k \mid k-1}}^{\star}+\sum_{j=k_{o}}^{k-1} \Delta \mathbf{p}_{R_{j}}^{\star}
\end{aligned}
$$

This completes the proof.

\section{ACKNOWLEDGEMENTS}

This work was supported by the University of Minnesota (DTC), and the National Science Foundation (IIS-0835637, IIS-0643680, IIS-0811946). Anastasios Mourikis was supported by the UMN Doctoral Dissertation Fellowship and the University of California, Riverside (BCOE).

\section{REFERENCES}

[Andrade-Cetto and Sanfeliu, 2004] Andrade-Cetto, J. and Sanfeliu, A. (2004). The effects of partial observability in SLAM. In Proc. IEEE International Conference on Robotics and Automation (ICRA), pages 394402, New Orleans, LA.

[Andrade-Cetto and Sanfeliu, 2005] Andrade-Cetto, J. and Sanfeliu, A. (2005). The effects of partial observability when building fully correlated maps. IEEE Transactions on Robotics, 21(4):771-777.

[Bailey et al., 2006] Bailey, T., Nieto, J., Guivant, J., Stevens, M., and Nebot, E. (2006). Consistency of the EKF-SLAM algorithm. In Proc. IEEE/RSJ International Conference on Intelligent Robots and Systems (IROS), pages 3562-3568, Beijing, China.

[Bar-Shalom et al., 2001] Bar-Shalom, Y., Li, X. R., and Kirubarajan, T. (2001). Estimation with applications to tracking and navigation. New York: Wiley.

[Castellanos et al., 2004] Castellanos, J., Neira, J., and Tardos, J. (2004). Limits to the consistency of EKF-based SLAM. In Proc. 5th IFAC Symposium on Intelligent Autonomous Vehicles, pages 1244-1249, Lisbon, Portugal.

[Castellanos et al., 2007] Castellanos, J. A., Martinez-Cantin, R., Tardos, J., and Neira, J. (2007). Robocentric map joining: Improving the consistency of EKF-SLAM. Robotics and Autonomous Systems, 55(1):21-29.

[Chen et al., 1990] Chen, Z., Jiang, K., and Hung, J. C. (1990). Local observability matrix and its application to observability analyses. In Proc. 16th Annual Conference of IEEE (IECON), pages 100-103, Pacific Grove, CA.

[Cover and Thomas, 1991] Cover, T. M. and Thomas, J. A. (1991). Elements of Information Theory. Wiley, New York.

[Goshen-Meskin and Bar-Itzhack, 1992] Goshen-Meskin, D. and BarItzhack, I. (1992). Observability analysis of piece-wise constant systems Part I: Theory. IEEE Transactions on Aerospace and Electronics Systems, 28(4):1056-1067.

[Hermann and Krener, 1977] Hermann, R. and Krener, A. (1977). Nonlinear controllability and observability. IEEE Transactions on Automatic Control, 22(5):728 - 740.

[Huang et al., 2008a] Huang, G. P., Mourikis, A. I., and Roumeliotis, S. I. (2008a). Analysis and improvement of the consistency of extended Kalman filter-based SLAM. In Proc. IEEE International Conference on Robotics and Automation (ICRA), pages 473-479, Pasadena, CA. 
[Huang et al., 2008b] Huang, G. P., Mourikis, A. I., and Roumeliotis, S. I. (2008b). A first-estimates Jacobian EKF for improving SLAM consistency. In Proc. 11th International Symposium on Experimental Robotics (ISER), Athens, Greece.

[Huang et al., 2008c] Huang, G. P., Mourikis, A. I., and Roumeliotis, S. I. (2008c). Generalized analysis and improvement of the consistency for EKF-based SLAM. Technical report, MARS Lab, University of Minnesota, Minneapolis, MN. www.cs.umn.edu/ ghuang/paper/TR_slam_genconsistency.pdf.

[Huang and Dissanayake, 2006] Huang, S. and Dissanayake, G. (2006). Convergence analysis for extended Kalman filter based SLAM. In Proc. IEEE International Conference on Robotics and Automation (ICRA), pages 412-417, Orlando, FL.

[Huang and Dissanayake, 2007] Huang, S. and Dissanayake, G. (2007). Convergence and consistency analysis for extended Kalman filter based SLAM. IEEE Transactions on Robotics, 23(5):1036-1049.

[Julier and Uhlmann, 2001] Julier, S. and Uhlmann, J. (2001). A counter example to the theory of simultaneous localization and map building. In Proc. IEEE International Conference on Robotics and Automation (ICRA), pages 4238-4243, Seoul, Korea.

[Kim and Sukkarieh, 2003] Kim, J. H. and Sukkarieh, S. (2003). Airborne simultaneous localisation and map building. In Proc. IEEE International Conference on Robotics and Automation (ICRA), pages 406-411, Taipei, Taiwan.

[Lee et al., 2006] Lee, K. W., Wijesoma, W. S., and Guzman, J. I. (2006). On the observability and observability analysis of SLAM. In Proc. IEEE/RSJ Intenational Conference on Intelligent Robots and Systems (IROS), pages 3569-3574, Beijing, China.

[Leonard et al., 2002] Leonard, J. J., Rikoski, R. J., Newman, P. M., and Bosse, M. (2002). Mapping partially observable features from multiple uncertain vantage points. International Journal of Robotics Research, 21(10-11):943-976.

[Meyer, 2001] Meyer, C. (2001). Matrix Analysis and Applied Linear Algebra. SIAM.

[Montemerlo, 2003] Montemerlo, M. (2003). FastSLAM: A factored solution to the simultaneous localization and mapping problem with unknown data association. $\mathrm{PhD}$ thesis, Carnegie Mellon University.

[Neira and Tardos, 2001] Neira, J. and Tardos, J. (2001). Data association in stochastic mapping using the joint compatibility test. IEEE Transactions on Robotics and Automation, 17(6):890 - 897.

[Newman, 1999] Newman, P. M. (1999). On the structure and solution of the simultaneous localization and mapping problem. $\mathrm{PhD}$ thesis, University of Sydney.

[Paskin, 2002] Paskin, M. (2002). Thin Junction Tree Filters for Simultaneous Localization and Mapping. PhD thesis, UC Berkeley.

[Se et al., 2002] Se, S., Lowe, D., and Little, J. (2002). Mobile robot localization and mapping with uncertainty using scale-invariant visual landmarks. International Journal of Robotics Research, 21(8):735 - 758.

[Smith and Cheeseman, 1987] Smith, R. and Cheeseman, P. (1987). On the representation and estimation of spatial uncertainty. International Journal of Robotics Research, 5(4):56-68.

[Vidal-Calleja et al., 2007] Vidal-Calleja, T., Bryson, M., Sukkarieh, S., Sanfeliu, A., and Andrade-Cetto, J. (2007). On the observability of bearing only SLAM. In Proc. IEEE International Conference on Robotics and Automation (ICRA), pages 4114-4118, Roma, Italy.

[Williams et al., 2000] Williams, S. B., Newman, P. M., Dissanayake, M., and Durrant-Whyte, H. (2000). Autonomous underwater simultaneous localisation and map building. In Proc. IEEE International Conference on Robotics and Automation (ICRA), pages 1793-1798, San Francisco, CA. 